\title{
Price Competition in Product Variety Networks*
}

\author{
Philip Ushchev ${ }^{\dagger} \quad$ Yves Zenou $^{\ddagger}$
}

March 19, 2018

\begin{abstract}
We develop a product-differentiated model where the product space is a network defined as a set of varieties (nodes) linked by their degrees of substituability (edges). We also locate consumers into this network, so that the location of each consumer (node) corresponds to her "ideal" variety. We show that there exists a unique Bertrand-Nash equilibrium where prices are determined by both the firms' sign-alternating Bonacich centralities and the average willingness to pay across consumers. We also investigate how local product differentiation and the spatial discount factor affect the equilibrium prices. We show that these effects non-trivially depend on the network structure. In particular, we find that, in a star-shaped network, the central firm does not always enjoy higher monopoly power than the peripheral firms.
\end{abstract}

Keywords: networks, product variety, monopolistic competition, spatial competition. JEL Classification: D43, L11, L13.

${ }^{*}$ We are grateful to the editor and two anonymous referees for helpful comments. We owe special thanks to J.-F. Thisse for detailed and useful discussions. We are also grateful to K. Behrens, S. Dhingra, G. Felbermayr, V. Ivanova, S. Kokovin, S. Krautheim, A. Kushnir, Y. Murata, M. Parenti, M. Sandomirskaya, and A. Tarasov for comments and suggestions. The study has been funded by the Russian Academic Excellence Project '5-100'.

${ }^{\dagger}$ National Research University Higher School of Economics, Russian Federation. Email: ph.ushchev@gmail.com.

${ }^{\ddagger}$ Monash University, Southampton University and IFN. E-mail: yves.zenou@monash.edu. 


\section{Introduction}

Economists have followed Lancaster $(1966,1979,1990)$ in his description of differentiated products, where a product is defined as a bundle of $n$ characteristics and is visually represented by a point in an $n$-dimensional Euclidean space. However, even though Euclidean geometry is intuitive and familiar, its representation is far from being innocuous. For instance, as argued by neuroeconomists, the brain encodes images of products in neural networks that may have a complex structure (Camerer et al., 2005; Glimcher and Fehr, 2013). The main message of this paper is that modeling product differentiation using networks rather than Euclidean spaces has dramatic and unsuspected consequences for the study of a differentiated industry.

Our point of departure is that understanding the nature of market competition requires a flexible tool that maps the fundamental features of the product space into substitutability patterns across varieties. Our setting captures the following two facets of the demand side: (i) proximity of each variety to a consumer's most preferred variety, and (ii) the binary relationship of direct substitutability between varieties. This is achieved by making a distinction between product differentiation and market segmentation.

Indeed, the concepts of product differentiation and market segmentation are both of paramount importance for research in industrial economics and related fields. At the same time, the marketing literature has been repeatedly raising concerns about the lack of precision in defining these concepts and separating them from each other. As stated by Dickson and Ginter (1987), "despite the pervasive use of the terms "market segmentation" and "product differentiation", there has been and continues to be considerable misunderstanding about their meaning and use". In this paper, we make a theoretical attempt to separate product differentiation from market segmentation. To the best of our knowledge, we are the first to do so from an economic theory perspective 1

To achieve this, we propose a model of imperfect competition in which consumers are heterogeneous and have linear-quadratic preferences. Each consumer has a "most-preferred" variety, for which her willingness to pay is higher than for any other variety. This is where consumer heterogeneity stems from: different consumers have different most-preferred varieties. Therefore, we are in an horizontal product differentiation framework and not a vertical one, since there is no unique ordering over the set of varieties, like in, for example, Gabszewicz and Thisse (1979). However, our setting also differs from a discrete-choice environment used by Hotelling (1929) and Salop (1979) since, in our model, consumers purchase in volume and they may choose to purchase more than one variety. Furthermore, we link the willingness-to-pay of a particular consumer for a particular variety to the geodesic distance between this variety and her ideal variety in a product space.

The distinction between product differentiation and market segmentation is captured as follows. The degree of product differentiation is measured by the cross-term coefficient in the linearquadratic utility function (which is assumed to be the same across consumers), while the structure of market segmentation is described by a network: there is a link between two firms if and only if they belong to a common market segment. Because this concept is somewhat elusive, market segments are difficult to identify. If one could define it in an unambiguous way, a market segmen-

\footnotetext{
${ }^{1}$ The approaches proposed in the marketing literature (e.g. Dikson and Ginter, 1987; Kamakura and Russell, 1989) suffer from lack of microfoundations.
} 
tation would always be given by a partition of a set of firms: each firm would certainly belong to one and only one segment of the market. A network representing this type of relationship would include several connected components, each of them being a complete graph. However, market areas targeted by different sets of firms may overlap. In other words, if firms $A$ and $B$ share a common market segment while firms $B$ and $C$ also share a common market segment, it does not have to be true that firms $A$ and $C$ belong to a common segment 2 As a consequence, the network structures generated by market segmentations may be much more complex than just a collection of complete networks. We call these network structures product-variety networks.

Let us illustrate this idea with Figure 1 where there are 11 firms and 5 market segments, and these segments overlap. We say that two firms are linked if they share at least one segment. The resulting network structure is more sophisticated than that of a complete graph. For example, consider the largest circle in the left panel of Figure 1, which includes 5 firms. This is a segment of the market and we see, in the right panel of Figure 1 that all these 5 firms are linked to each other in the network. In other words, within a segment, all firms are linked to each other because their products share the same characteristics, i.e. they are all direct substitutes. In other words, there is a one-to-one relationship between market segments and network structure. We show below that this structure, in turn, implies a micro-founded centrality measure, which is the sign-alternating Bonacich centrality with specific weights. Therefore, for each individual firm, what matters is how the whole market is segmented (i.e. what is the "global"structure of the market) and not just the behavior of the closest competitors (i.e. firms belonging to the same market segment).

Figure 1: Market segmentations and product variety networks

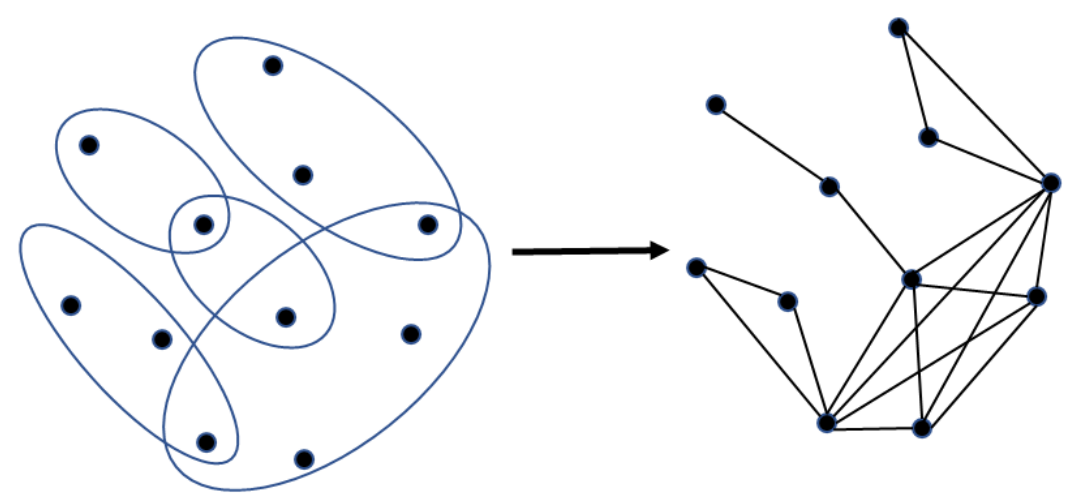

Thus, by defining different segments for different firms, we are able to determine a productvariety network. However, if we want to empirically define a product-variety network, the task is complicated. In Appendix B, we provide an emprical link between market segmentation and product-variety network through the characteristic space of the products. Basically, we say that two firms share the same market segment if and only if the characteristics of products they supply are "sufficiently" close to each other

\footnotetext{
${ }^{2}$ Formally, the relationship " $A$ and $B$ share a common market segment" need not to be transitive.
} 
It is, however, very difficult to empirically define the "proximity" of two products or even the boundries of an industry (see e.g. Einav and Levin, 2010). Recently, Hoberg and Phillips (2016) have proposed a new way of defining this proximity, which is based on the notion that firms in the same industry use many of the same words to identify and describe their products. Their approach can then identify competitors of each firm on the basis of the similarity of their product offerings. Hoberg and Phillips (2016) are then able to define how similar each firm is to every other firm by calculating firm-by-firm pairwise word similarity scores. Using these pairwise similarity scores, they can group firms into industries. Their general industry classification can then be represented as a network of firms.

Our main findings can be summarized as follows. First, we fully characterize the BertrandNash equilibrium for any possible network. In particular, we show that the equilibrium price of any firm is a function of its sign-alternating Bonacich centrality (Bonacich, 1987) based on the premise that the node's importance is determined by how important its neighbors are. The degree of substitutability between the neighboring varieties serves as the discount factor for the "importance" of neighbors. The firm's equilibrium price also reflects the average willingness-topay across consumers, which, in turn, depends on the structure of the network. Interestingly, what matters for the characterization of the equilibrium outcome is a "signed" modification of the Bonacich centrality (where the discount factor changes sign with the distance in the network), rather than the standard Bonacich centrality with positive discount factor (Ballester et al., 2006). This feature of the model implies that prices are neither strategic complements nor strategic substitutes, except for the special case of a complete network. In other words, "my enemy's enemy is my friend". This result highlights the difference between our model and the standard differentiated Bertrand settings, where prices are typically strategic complements (Vives, 1999). Furthermore, we find that the (sign-alternating) Bonacich centrality matrix is the Slutsky matrix associated with the demand system generated by the underlying product-variety network.

Second, we investigate how the degree of substitutability between neighboring varieties affects the equilibrium prices. When products are highly differentiated, a small drop in the degree of differentiation makes competition tougher and reduces all prices. However, the magnitude of price reduction depends on both the network structure and the distance decay factor. If, for example, we consider a star-shaped network, we find that the firm located in the star node needs not enjoy higher monopoly power than the other firms. This is because the firm located in the center has better access to the market than the peripheral firms, but it also competes with all peripheral firms. Which of the two effects prevails depends on the value of the degree of substitutability across varieties.

Third, we study how the spatial discount factor, which captures the fact that there is an "exponential decay" effect with respect to distance, affects the equilibrium prices. In particular, we show that, when products are highly differentiated, an increase in the spatial discount factor leads to higher prices. The intuition behind this result is easy to grasp: when this factor increases, each firm has less monopoly power over consumers situated in the close neighborhood, but a better access to the distant consumers. The latter effect dominates the former when varieties are "bad" substitutes. On the contrary, when varieties are close substitutes, the opposite may occur, for which we provide examples obtained via simulations. 
We also investigate the special case of regular networks and provide a necessary and sufficient condition for a symmetric equilibrium to exist. In this case, we show that less localized competition or denser networks, i.e. more links in the network with the same number of nodes, may lead to either lower or higher equilibrium prices. Two effects are at work: the competition effect, which reflects an increase in the toughness of competition when the network gets more connected, and the market access effect, which is due to the fact that new links bring all consumers closer to each firm. The market access effect drives prices upwards, while the competition effect leads to a reduction in prices. We show that a lower bound and an upper bound of the spatial discount factor exist, such that the competition effect dominates the market access effect if and only if the spatial discount factor is within the two bounds. Otherwise, the result is reversed.

Finally, we discuss the welfare implications of our model. First, we provide a simple expression of the total welfare. Second, even though there are externalities, we show that the welfare is maximized when prices are competitive. Finally, we study the welfare consequences of an increase in the density of the product-variety network.

The rest of the paper unfolds as follows. In the next section, we review the related literature and highlight our contribution. In Section 3, we describe our model and determine the Bertrand-Nash equilibrium. We perform the comparative statics exercises of our model in Section 4. Symmetric equilibria are analyzed in Section 5. The welfare effects of our model are investigated in Section 6 . Section 7 concludes. All proofs can be found in Appendix A.

\section{Related literature}

In industrial organization, there are two dominating approaches to modeling product differentiation: (i) spatial competition, also known as the address approach, which was first suggested by Hotelling (1929) and further developed by Lancaster (1966), and (ii) monopolistic competition, introduced by Chamberlin (1933) and formalized by Spence (1976) and Dixit and Stiglitz (1977) $\mathrm{u}^{3}$

Each of these approaches has generated a large flow of contributions, which have been applied to a wide range of economic issues (see, e.g. Helpman and Krugman, 1989; Fujita and Thisse, 2013). However, both approaches have limitations. On the one hand, spatial competition a la Hotelling relies on the principle of mutually exclusive choices, meaning that each consumer purchases only one variety. On the other hand, all varieties are assumed to be equally good substitutes in models of monopolistic competition and due to consumers' love for variety, all varieties are consumed in equal volumes. As a consequence, the two frameworks feature strong dissimilarities in patterns of consumers' behavior, which, in turn, imply different properties of market outcomes. In this paper, we aim at providing a unifying framework for studying imperfect competition in a "firm-product" space, which would capture both features of Hotelling's and Chamberlin's models.

Our model can be viewed as a further development of both monopolistic competition models (Matsuyama, 1995) and the "spokes" model of product differentiation proposed by Chen and Riordan (2007), where the network represents the variety space. From the technical viewpoint, our approach is related to literature on games on networks (Jackson and Zenou, 2015) where the

\footnotetext{
${ }^{3}$ For overviews, see Anderson et al. (1992) and Matsuyama (1995).
} 
network is explicitly modeled as a graph and the payoff functions are linear-quadratic (see, in particular, Ballester et al., 2006; Bramoullé et al., 2014; Calvó-Armengol et al., 2009) ${ }^{4}$ The focus and results are, however, very different to ours. There is also a growing literature that models price competition between firms with an explicit network. Two important papers in this literature are that of Bloch and Quérou (2013) and Candogan et al. (2012) 5 Bloch and Quérou (2013) study optimal monopoly pricing in the presence of network externalities across consumers. The setting proposed by these authors involves a homogeneous good produced by a monopolist, and many consumers whose probability to purchase the good. Candogan et al. (2012) develop a similar approach, but with a divisible good 6 In contrast to these papers, we account for product differentiation and consider a price-setting game among several firms.77 There is also an interesting literature on more general aspect of industrial organization and networks. However, most papers in this literature (Goyal and Moraga-Gonzalez, 2001; Goyal and Joshi, 2003; Westbrock, 2010; König et al., 2018) introduce the network through R\&D collaborations. We believe we are the first to apply the toolkit of games on networks to modeling competition in product-variety space within an address approach 8

To be precise, the main novelty of our modeling strategy compared to the previous literature may be described as follows. First, the ideal variety of each consumer, or, equivalently, consumer's location in a "firm-product" space, is a node in the network. Second, the geodesic distance between nodes measures the degree of taste heterogeneity. In addition, the degree of pairwise substitutability between product varieties is high (low) - or, equivalently, firms are (are not) involved into headto-head competition - when there is a link (there is no link) between the corresponding nodes. In other words, the principal role of a network in our model is that it captures the substitutability relationship between differentiated products. This is where our work departs from the modern "non-spatial" paradigm of modeling imperfect competition in international trade (Ottaviano et al., 2002; Melitz, 2003; Melitz and Ottaviano, 2008; Dhingra, 2013; Mayer et al., 2014), where the substitution term is the same across varieties and where heterogeneities are mostly on the supply side 9 The relationship of our work to this strand of literature is best described as follows. Recent

\footnotetext{
${ }^{4}$ The economics of networks is a growing field. For overviews, see Jackson (2008, 2014), Bramoullé et al. (2016), and Jackson et al. (2017).

${ }^{5}$ See also Shi (2003), Deroian and Gannon (2006), Banerji and Dutta (2009), Billand et al. (2014), Carroni and Righi (2015), Currarini and Feri (2015) and Chen et al. (2018).

${ }^{6}$ See also Bimpikis et al. (2015) who develop a model with a bipartite graph where nodes are either firms or markets and a link between firm $i$ and market $j$ exists if firm $i$ operates in market $j$.

${ }^{7}$ There is also an interesting literature in operation research where the location problem is captured by more general topologies, such as networks. One of the first papers is that of Wendell and McKelvey (1981) who extend the basic model of Hotelling to a network context in which vertices represent potential products as well as consumers, and edges succinctly encode the possible assignments of consumers to products. A recent paper of this literature is that of Gur et al. (2017) who consider a competitive facility location problem on a network in which consumers located on vertices wish to connect to the nearest facility and each competitor locates a facility on a vertex trying to maximize her market share. This literature, however, mostly focuses on the location problem and takes prices as given.

${ }^{8}$ Note that Gabszewicz and Thisse (1986) have developed within spatial competition theory a graph-theoretic setup to determine rigorously the set of firms forming an industry in the spatial economy.

${ }^{9}$ In the modern trade literature, network perspective is typically used for studying free-trade agreements (see, e.g., Furusawa and Konishi, 2007). A notable exception is Behrens et al. (2007), who consider trading countries as nodes of a spatial network and stress the role of Bonacich centrality for understanding the equilibrium trade patterns.
} 
studies of monopolistic competition under variable elasticity of substitution (Behrens and Murata, 2007; Zhelobodko et al., 2012; Parenti et al., 2017) go in the direction of dealing with more and more general classes of symmetric consumers' utilities, remaining within the non-spatial paradigm. We, instead, choose to study the consequences of a non-specified network structure of the product space. This is done at the cost of working with a relatively specific family of utilities (namely, linear-quadratic), which are well known to be best suited for studying games on networks.

Finally, our paper echoes the logit model of product differentiation (Anderson et al., 1992; 1995), in which combining the ideal-variety and the love-for-variety approach is achieved by introducing a probabilistic choice on the consumers' side. Furthermore, our comparative statics results, while being generically different from those obtained within the standard spatial competition approach a la Hotelling (see Section 4.2), parallel recent findings by Chen and Riordan (2008) on price-increasing competition. We differ from all these authors by stressing the role of market segmentation, which we model by means of a network.

\section{The model}

In this section, we set our model of price competition in product-variety networks. Since we interpret commodities as varieties of a differentiated good, we find it natural to focus on the case when varieties are gross substitutes: a reduction in price for variety $i$ normally leads, other things being equal, to a reduction in the quantity demanded for variety $j$. This assumption holds in a number of models of product differentiation in industrial organization (see Anderson et al., 1992, for an extensive survey). Therefore, given the context, disregarding the case of gross complements seems to be quite natural. We also assume that each variety is someone's most preferred variety, and that every two consumers differ in their most preferred varieties. In other words, there is a one-to-one correspondence between varieties/nodes and consumers so that each node accommodates only one consumer. We keep this assumption for two reasons. First, it allows us to analyze the impact of the network structure on the market outcome, net of the effects of consumer's distribution across the product space. Second, a uniform distribution of consumers across the nodes is a natural counterpart of assuming a uniformly distributed population in the Hotelling linear city model. We also argue that this assumption does not crucially affect the nature of our results. See our comment after equation (17) in Section 3.2.3.

\subsection{Notations and definitions}

There are $N$ firms that produce $N$ different varieties, each firm $i=1, \ldots, N$ producing one variety $i$. Each firm/variety is embedded into a network $(\mathcal{N}, \mathbf{G})$, where each variety $i \in \mathcal{N} \equiv\{1,2, \ldots, N\}$ is a node, while $\mathbf{G}=\left(g_{i j}\right)_{i, j=1 \ldots N}$ is the adjacency matrix that keeps track of the degree of substitutability between the neighboring varieties in the network. To be more precise, there is a link (i.e. $\left.g_{i j}=1\right)$

Note also that Osharin et al. (2014) and Tarasov (2014) study income-taste heterogeneities across consumers within non-spatial settings. Their approaches, however, are substantially different from ours, for the demand side in their model is described by the standard CES utility, hence, any two varieties are equally substitutable. A setting closer to ours is used by Di Comite et al. (2014), who work with asymmetric linear-quadratic preferences to study the empirical implications of country-specific taste mismatch patterns on trade flows. 
between varieties $i$ and $j$ if and only if these two varieties are direct substitutes. Otherwise, a link does not exist, i.e. $g_{i j}=0$. By convention, $g_{i i}=0$. Quite naturally, $g_{i j}=g_{j i}$ so that the network is undirected, which implies that $\mathbf{G}$ is a square $(0,1)$ symmetric matrix with zeros on its diagonal.

We have the following standard network-related definitions. A walk in a network $(\mathcal{N}, \mathbf{G})$ refers to a sequence of nodes, $i_{1}, i_{2}, i_{3}, \ldots, i_{L-1}, i_{L}$ such that $g_{i_{l} i_{l+1}}=1$ for each $l$ from 1 to $L-1$. The length of the walk is the number $L-1$ of links in it. A path in a network $(\mathcal{N}, \mathbf{G})$ is a walk in $(\mathcal{N}, \mathbf{G}), i_{1}, i_{2}, i_{3}, \ldots, i_{K-1}, i_{K}$, such that all the nodes are distinct. The (geodesic) distance $d_{i j}$ between two nodes $i$ and $j$ in a network is the length of a shortest path between them. The $s$ th power $\mathbf{G}^{s}=\mathbf{G} \times{ }^{\text {(s times) }} \times \mathbf{G}$ of the adjacency matrix $\mathbf{G}$ keeps track of indirect connections in $\mathbf{G}$. More precisely, the coefficient $g_{i j}^{[s]}$ in the $(i, j)$ cell of $\mathbf{G}^{s}$ gives the number of walks of length $s$ in $\mathbf{G}$ between $i$ and $j$. The set of neighbors (here direct substitutes) of node (here variety) $i$ in network $(\mathcal{N}, \mathbf{G})$ are denoted by $\mathcal{N}_{i}=\left\{\right.$ all $\left.j \mid g_{i j}=1\right\}$.

In our model, the distance between two products in the network measures the degree of substitutability between these two products so that the higher is the distance, the poorer substitutes are these products. In other words, the network $(\mathcal{N}, \mathbf{G})$ plays the role of a "firm-product" space in the model and captures the degree of substitution between $N$ varieties supplied in the economy. Because we do not impose any specific assumptions about the network structure, we find that our approach is flexible enough to encompass different types of spatial structures, commonly studied in the industrial-organization literature. Figure 2 illustrates some of these networks for $N=4$. Going back to our discussion in the Introduction, the Chamberlin-type competition (due to Chamberlin, 1933), which corresponds to the complete network (Figure 2a), has one market segment, the Hotelling-type competition (due to Hotelling, 1929), which corresponds to the line or chain network (Figure 2b) has 3 market segments, the Salop-type competition (due to Salop, 1979), which is a circle network (Figure 2c), has four market segments and, finally, the Chen-Riordan-type competition (due to Chen and Riordan, 2007), which is a star network (Figure 2d), has three market segments.

As stressed in the Introduction, this setup brings together love for variety and spatial taste heterogeneity, while giving access to a rich array of research techniques developed recently in games on networks. An obvious critique to our approach is that the product space underlying our model is essentially discrete, while monopolistic competition models (starting with Dixit and Stiglitz, 1977) typically involve a continuum of varieties. We believe that this seemingly major discrepancy does not raise, in fact, any conceptual difficulty. Following the same lines as in Hart (1979), it is fairly straightforward to develop a "continuous" counterpart of our setting, in which the product-variety space could be represented by, say, a Riemannian manifold (or a more general compact metric space). We choose, however, to work instead with networks, for two reasons. First, we believe that they are more intuitive objects for most economists. Second, we find it more natural to build on the toolkit of games on networks rather than to develop from scratch conceptually similar techniques for general compact metric spaces. 
Figure 2: Some commonly used product-variety networks

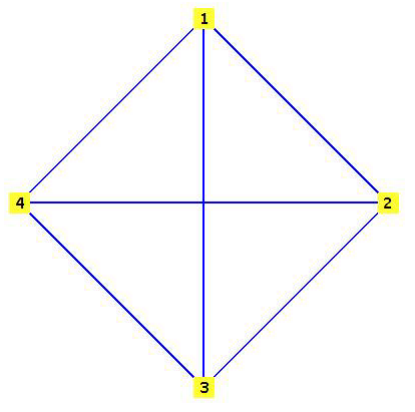

2.a. Chamberlin-type competition: Complete network

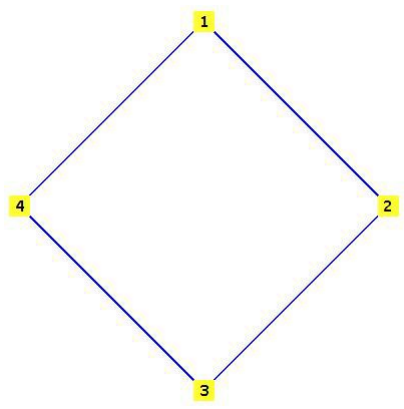

2.c. Salop-type competition: Cycle network

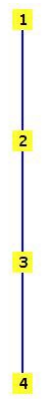

2.b. Hotelling-type competition: Chain network

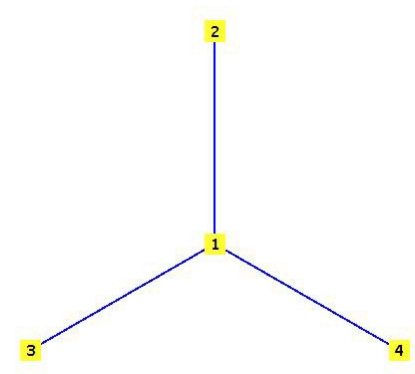

2.d. Chen-Riordan-type competition: Star network 


\subsection{Consumers}

We have seen that a network is composed of varieties (nodes) linked by their degrees of substitutability (edges). In the network, we can also locate consumers so that the location of each consumer $k=1, \ldots, N$ corresponds to her "ideal" variety. As a result, there are as many consumers as varieties.

\subsubsection{Preferences}

A consumer located at location/node $k \in \mathcal{N}$, i.e. whose ideal variety is $k$, has the following linear-quadratic utility function:

$$
U(k, \mathbf{G})=x_{0 k}+\sum_{i \in \mathcal{N}} \alpha_{i k} x_{i k}-\frac{\beta}{2} \sum_{i \in \mathcal{N}} x_{i k}^{2}-\frac{1}{2} \sum_{i, j \in \mathcal{N}} \gamma_{i j} x_{i k} x_{j k}
$$

where $x_{0 k}$ is the level of consumption of an outside good, $x_{i k}$ is the volume of consumer $k$ 's purchases of variety produced by firm $i$ (i.e. located at node $i$ ) whereas $\alpha_{i k}$ is the consumer-specific willingness to pay for variety $i$. The term $\frac{\beta}{2} \sum_{i \in \mathcal{N}} x_{i k}^{2}$ accounts for the consumer's love for variety via the parameter $\beta>0$. This term does not depend on the network structure and is referred to as the love-for-variety effect. Finally, the cross-term $\sum_{i, j \in \mathcal{N}} \gamma_{i j} x_{i k} x_{j k}$ captures the substitutability linkages across varieties and is referred to as the substitution effect.

Assuming linear-quadratic preferences is natural for two reasons. First, ever since Singh and Vives (1984), it has been a workhorse model in differentiated oligopoly theory (see Belleflamme and Peitz, 2010, Ch. 3). Second, at least since Ottaviano et al. (2002), symmetric linear-quadratic preferences have been widely used in trade literature (Melitz and Ottaviano, 2008) and urban economics literature (Picard and Tabuchi, 2013). We differ from all these authors in two respects, since we work with preferences that are asymmetric both across varieties, like in Foster et al. (2008) or Picard and Okubo (2012), and heterogeneous across consumers, like in Di Comite et al. (2014).

To be precise, we assume that the parameters $\alpha_{i k}$ and $\gamma_{i j}$ depend on the product-variety network structure. In particular, following Jackson and Wolinsky (1996), we assume that $\alpha_{i k}$ is a decreasing function of the (geodesic) distance $d_{i k}$ in the network between variety $i$ and the ideal variety of consumer $k$ :

$$
\alpha_{i k}=\alpha \phi^{d_{i k}}
$$

where $0<\phi<1$ is a spatial discount factor. This captures the fact that there is an "exponential decay" of the attractiveness of varieties with distance. Observe that the term $\sum_{i \in \mathcal{N}} \alpha_{i k} x_{i k}$ captures the proximity of other varieties to the ideal variety of consumer $k$ and depends on the location of $k$ in the network (i.e. her ideal product). It is referred to as the proximity network effect.

Furthermore, we assume that $\gamma_{i j} \equiv \gamma g_{i j}$ with $\gamma>0$, so that only direct substitutes for which $g_{i j}=1$ have an impact on the marginal utility of consuming variety $i$. As discussed in the Introduction, the parameter $\gamma$ is an inverse measure of product differentiation, while the network $g_{i j}$ keeps track of market segmentation. A higher $\gamma$ means that varieties are less differentiated and thus the consumption of close substitutes reduces the utility of consuming variety $i$.

Note that a necessary and sufficient condition for consumers to have love for variety (or, more 
formally, to have strictly convex preferences over the space of differentiated products) is that $\beta / \gamma>-\lambda_{\min }(\mathbf{G})$, where $\lambda_{\min }(\mathbf{G})$ is the smallest eigenvalue of the adjacency matrix $\mathbf{G}$. This condition will be made more precise in what follows (see condition (4) in Lemma 1 below).

At this stage, we find it useful to compare our setting to the standard approaches in product differentiation models (Belleflamme and Peitz, 2010, Chap. 5). On the one hand, in love-for-variety models of market competition (monopolistic competition) with linear-quadratic utility, $\gamma$ serves as the sole (inverse) measure of product differentiation while $\alpha$ is not individual-specific. On the other hand, in spatial competition models, the transportation cost is a measure of product differentiation. Our setting differs from both these frameworks in at least two respects. First, instead of $\alpha$, we have here $\alpha_{i k}$, defined by (2), which depends on the location of the consumer $k$ in the network, while the spatial discount factor $\phi$ is the counterpart of the transportation cost in models a la Hotelling. Second, instead of $\gamma$, we have $\gamma_{i j} \equiv \gamma g_{i j}$, which depends on the structure of the network. To sum up, we have an essentially multidimensional description of how differentiated varieties are, given by $\phi, \gamma$, and the network G. Indeed, $\phi$ keeps track of the degree to which a consumer's valuation of the ideal variety exceeds that of any other variety, $\gamma$ measures the degree of substitutability, $\beta$ measures the degree of product differentiation, and $\mathbf{G}$ captures market segmentation.

Observe that, when $\phi=1$, then no consumer has ideal variety. If, in addition, the network is complete (Chamberlin network, Figure 2a), we are back to the standard representative consumer's approach, where only the love for-variety effect matters, while the proximity network effect does not. Conversely, when $\phi=0$, then consumer $k$ 's willingness to pay for any differentiated variety $i \neq k$ is clearly zero. In this limiting case, things work as if consumer $k$ 's preferences were defined solely over two goods, $z$ and $x_{k}$, and were given by $U_{k}\left(z, x_{k}\right)=z+\alpha x_{k}-\beta x_{k}^{2} / 2$. Hence, when $\phi=0$, the network structure plays no role, and the market demand for variety $i$ coincides with the individual demand of consumer $k=i$ for this variety. Let us now study the most interesting case, $\phi \in(0,1)$, in which the demand structure is more involved.

\subsubsection{Individual demand}

For the sake of the exposition, we rewrite the utility function (1) in vector-matrix form:

$$
U(k, \mathbf{G})=x_{0 k}+\boldsymbol{\alpha}_{k}^{T}(\phi, \boldsymbol{G}) \mathbf{x}_{k}-\frac{\beta}{2} \mathbf{x}_{k}^{T}(\mathbf{I}+\delta \boldsymbol{G}) \mathbf{x}_{k},
$$

where $\delta \equiv \gamma / \beta$ is a rescaled substitutability parameter, $\boldsymbol{\alpha}_{k}(\phi, \boldsymbol{G}) \equiv\left(\alpha_{i k}\right)_{i=1 \ldots N}$ and $\mathbf{x}_{k} \equiv\left(x_{i k}\right)_{i=1 \ldots N}$ are $N \times 1$ vectors, $\mathbf{I}$ is the $N \times N$ identity matrix and $\mathbf{x}^{T}$ is the transpose vector of $\mathbf{x}$. Denote by $\lambda_{1}(\mathbf{G}), \ldots, \lambda_{N}(\mathbf{G})$, the eigenvalues of $\boldsymbol{G}$ where, without loss of generality, $\lambda_{1}(\mathbf{G}) \geq \lambda_{2}(\mathbf{G}) \geq \ldots \geq$ $\lambda_{N}(\mathbf{G})$, so that $\lambda_{1}(\mathbf{G})$ is the largest eigenvalue of $\mathbf{G}$ while $\lambda_{N}(\mathbf{G})$ is the lowest eigenvalue of $\mathbf{G}$.

Lemma 1. The utility function (3) is strictly concave in $\mathbf{x}_{k}$ if and only if:

$$
\delta<-\frac{1}{\lambda_{N}(\mathbf{G})}
$$

Consumer $k$ seeks to maximize her utility (3) with respect to $\left(x_{0}, \mathbf{x}\right)$ subject to the budget constraint: 


$$
x_{0 k}+\mathbf{p}^{T} \mathbf{x}_{k}=Y_{k},
$$

where $Y_{k}$ is consumer $k$ 's income whereas $\mathbf{p}=\left(p_{i}\right)_{i \in \mathcal{N}}$ is the price vector. Plugging the value of $x_{0 k}$ from this budget constraint into (3) yields:

$$
U(k, \mathbf{G})=Y_{k}-\mathbf{p}^{T} \mathbf{x}_{k}+\boldsymbol{\alpha}_{k}^{T}(\phi, \boldsymbol{G}) \mathbf{x}_{k}-\frac{\beta}{2} \mathbf{x}_{k}^{T}(\mathbf{I}+\delta \boldsymbol{G}) \mathbf{x}_{k}
$$

The inverse demand of consumer $k$ for variety $i \in \mathcal{N}$ is given by:

$$
p_{i}=\alpha_{i k}(\phi, \boldsymbol{G})-\beta\left(x_{i k}-\delta \sum_{j \neq i} g_{i j} x_{j k}\right), i=1, \ldots, N
$$

or, in vector-matrix form,

$$
\mathbf{p}=\boldsymbol{\alpha}_{k}(\phi, \boldsymbol{G})-\beta(\mathbf{I}+\delta \boldsymbol{G}) \mathbf{x}_{k} .
$$

By solving (6) for $\mathbf{x}_{k}$, we obtain consumer $k$ 's individual demands for all varieties:

$$
\mathbf{x}_{k}^{*}=\frac{1}{\beta} \mathbf{B}(-\delta, \boldsymbol{G})\left[\boldsymbol{\alpha}_{k}(\phi, \boldsymbol{G})-\mathbf{p}\right],
$$

where $\mathbf{B}(-\delta, \boldsymbol{G}) \equiv(\mathbf{I}+\delta \boldsymbol{G})^{-1}$. Note that (4) implies that $\mathbf{B}$ is well-defined 10 Moreover, if

$$
\delta<\frac{1}{\lambda_{1}(\mathbf{G})}
$$

then $\mathbf{B}$ can be expanded in a power series as follows 11

$$
\mathbf{B}(-\delta, \boldsymbol{G})=\mathbf{I}-\delta \boldsymbol{G}+\delta^{2} \boldsymbol{G}^{2}-\delta^{3} \boldsymbol{G}^{3}+\ldots
$$

Following Bonacich (1987) and using (9), we may define the vector of sign-alternating Bonacich centrality measures of varieties as:

$$
\mathbf{b}(-\delta, \boldsymbol{G}, \mathbf{u}) \equiv \mathbf{B}(-\delta, \boldsymbol{G}) \mathbf{u}
$$

where $\mathbf{u}$ is any $N \times 1$ vector. We have: $b_{i j}=\sum_{s=0}^{\infty}(-\delta)^{s} g_{i j}^{[s]}$, or equivalently

\footnotetext{
${ }^{10}$ It is easily verified that if condition $\sqrt{8}$ is satisfied, then condition $(4)$ in Lemma $\sqrt{1}$, which guarantees that the utility function is strictly concave, is also satisfied. Indeed, since $\mathbf{G}$ is symmetric, then all its eigenvalues are real. Furthermore, we have that, by definition, $\left|\lambda_{1}(\mathbf{G})\right|>\left|\lambda_{N}(\mathbf{G})\right|$. Condition (4) can be written as: $-\delta \lambda_{N}(\mathbf{G})<1($ note that $\lambda_{N}(\mathbf{G})<0$; see footnote 17] while condition (8) can be written as: $\delta \lambda_{1}(\mathbf{G})<1$. Since $\delta \lambda_{1}(\mathbf{G})>-\delta \lambda_{N}(\mathbf{G})$, the result follows.

${ }^{11}$ For example, under Hotelling competition (Figure 2b) with $N$ varieties, we have $N$ distinct eigenvalues and the largest eigenvalue $\lambda_{1}(\mathbf{G})$ of this matrix is less than 2 (but converges to 2 when $N \rightarrow \infty$ ). Moreover, if $\lambda$ is an eigenvalue of $\mathbf{G}$, then $-\lambda$ is also an eigenvalue of $\mathbf{G}$. Hence, a sufficient condition for (4) and (8) to hold is: $\gamma \leq 1 / 2$. Under Salop competition (Figure 2c) with $N$ varieties, the largest eigenvalue $\lambda_{1}(\mathbf{G})$ of this matrix is equal to 2 . Hence, (4) and (8) boil down to $\gamma<1 / 2$.
} 


$$
b_{i j}=\left\{\begin{array}{cc}
1+\delta^{2} g_{i i}^{[2]}-\delta^{3} g_{i i}^{[3]}+\ldots & \text { for } i=j \\
-\delta g_{i j}+\delta^{2} g_{i j}^{[2]}-\delta^{3} g_{i j}^{[3]}+\ldots & \text { for } i \neq j
\end{array}\right.
$$

Observe that $g_{i j}^{[s]}$ is the number of walks of length $s$ in the network, which starts at variety $i$ and ends at variety $j$ (see Section 3.1). In particular, $g_{i i}^{[2]}$ is just the number of neighbors (i.e. direct substitutes) of variety $i$ while $g_{i i}^{[3]}$ is the number of triangles involving $i$, i.e. the number of couples of direct substitutes, which are also substitutes for each other. As can be seen from (11), $b_{i i}$ can be viewed as a firm-specific measure for toughness of competition faced by a firm producing variety $i$. When firm $i$ 's closest competitors also compete with each other, this relaxes the burden of competition borne by firm $i$. That is why $g_{i i}^{[3]}$ enters 11 with the negative coefficient $(-\delta)^{3}$. The cycles of higher orders are also taken into account, but their weight decays exponentially with $s$.

Two comments are in order. First, $\mathbf{B}(-\delta, \boldsymbol{G})$ has a clear interpretation: it is the Slutsky matrix associated with the demand system (7). Indeed, consider a vector dp of price changes. Then, consumer $k$ 's individual demands respond to these changes are as follows:

$$
\mathrm{d} \mathbf{x}_{k}^{*}=-\frac{1}{\beta} \mathbf{B}(-\delta, \boldsymbol{G}) \mathrm{d} \mathbf{p}=-\frac{1}{\beta} \mathbf{b}(-\delta, \boldsymbol{G}, \mathrm{d} \mathbf{p}) .
$$

In other words, a variety that has a higher (lower) centrality in the product-variety network means that the demand for this variety is more (less) sensitive to changes in prices.

Second, in the literature on games on networks (Jackson and Zenou, 2015), the Bonacich centrality is usually defined as $\mathbf{B}(\delta, \boldsymbol{G}) \mathbf{u}$ where

$$
\mathbf{B}(\delta, \boldsymbol{G})=\mathbf{I}+\delta \boldsymbol{G}+\delta^{2} \boldsymbol{G}^{2}+\delta^{3} \boldsymbol{G}^{3}+\ldots
$$

has no negative terms. Here, we have a different definition (also considered in Bonacich, 1987): even powers of $\mathbf{G}$ are weighted positively and odd powers negatively. As a result, having many direct ties (degree) reduces centrality, but, if one's connections themselves have many connections, centrality is augmented. This property of our model is key since it drives the "my enemy's enemy is my friend" result. Indeed, restate (7) as follows:

$$
x_{i k}^{*}=\frac{1}{\beta}\left[\sum_{j \in \mathcal{N}} b_{i j} \alpha_{j k}(\phi, \boldsymbol{G})-b_{i i} p_{i}-\sum_{j \in \mathcal{N}, i \neq j} b_{i j} p_{j}\right]
$$

The individual demand (14) of consumer $k$ for variety $i$ is made of three terms. First, the intercept of the individual demand, $\frac{1}{\beta} \sum_{j \in \mathcal{N}} b_{i j} \alpha_{j k}(\phi, \boldsymbol{G})$, shows the saturation level. Observe that the intercept of the individual demand is the only part of the demand function which is individual specific and depends of the consumer $k$ 's position in the network. Second, the own price effect, $\frac{1}{\beta} b_{i i} p_{i}$, captures the effect of price of variety $i$ on its own demand. The marginal impact of the price $p_{i}$ on demand $x_{i k}^{*}$ is equal to $b_{i i}$, which is, by (11), the discounted number of cycles involving $i$. Thus, $b_{i i}$ is network 
specific, but not individual specific. Finally, $\frac{1}{\beta} \sum_{j \in \mathcal{N}, i \neq j} b_{i j} p_{j}$, corresponds to the cross-price effects, which clearly depend on the structure of the network via $b_{i j}$. For instance, a complete network will generate price effects very different from those in a star network. In the Online Appendix 1, we illustrate these differences by comparing the Chamberlin-type spatial structure (Figure 2a) and the Chen-Riordan spatial structure (Figure 2d).

\subsubsection{Aggregate demand}

Using (7), the vector $\mathbf{X}^{*} \equiv \sum_{k \in \mathcal{N}} \mathbf{x}_{k}^{*}$ of aggregate demands faced by firms is given by:

$$
\mathbf{X}^{*}=\frac{1}{\beta} \mathbf{B}(-\delta, \boldsymbol{G})\left[\sum_{k \in \mathcal{N}}\left(\boldsymbol{\alpha}_{k}(\phi, \boldsymbol{G})-\mathbf{p}\right)\right] .
$$

Define the vector of average willingness to pay across consumers as

$$
\overline{\boldsymbol{\alpha}}(\phi, \boldsymbol{G}) \equiv \frac{1}{N} \sum_{k \in \mathcal{N}} \boldsymbol{\alpha}_{k}(\phi, \boldsymbol{G}) .
$$

Since $\alpha_{i k}=\alpha \phi^{d_{i k}}$, we have:

$$
\bar{\alpha}_{i}(\phi, \boldsymbol{G})=\frac{\alpha}{N}\left[1+\phi N_{1}(i)+\phi^{2} N_{2}(i)+\ldots+\phi^{d} N_{d}(i)\right],
$$

where $d \equiv \max _{i, k \in \mathcal{N}} d_{i k}$ is the diameter of the network while $N_{l}(i)$ is the number of nodes (varieties) whose geodesic distance from node $i$ equals $l$. In particular, $N_{1}(i)$ is the degree of node $i$. Using (16) and (15), we can write aggregate demand for variety $i$ as follows:

$$
X_{i}^{*}=\frac{N}{\beta}\left(\sum_{j \in \mathcal{N}} b_{i j} \bar{\alpha}_{j}-b_{i i} p_{i}-\sum_{j \neq i} b_{i j} p_{j}\right),
$$

where $b_{i j}$ are the elements of $\mathbf{B} \equiv \mathbf{B}(-\gamma, \boldsymbol{G})$ (see $(11)$ ). In order to guarantee that expressions (17) make economic sense, we have to require that the choke-off price of aggregate demand for each variety is positive. In other words, the following inequalities must hold:

$$
\sum_{j \in \mathcal{N}} b_{i j} \bar{\alpha}_{j}>0 \quad \text { for all } i \in \mathcal{N} .
$$

Observe that (18) always holds when $\delta$ is not too large, i.e. when products are sufficiently differentiated. Indeed, as implied by (11), we have:

$$
\lim _{\delta \rightarrow 0} b_{i j}= \begin{cases}1, & \text { for } i=j \\ 0, & \text { for } i \neq j\end{cases}
$$


A sufficient condition for 18 to hold is that $\mathbf{B}$ is a strictly diagonally dominant matrix with weights $\bar{\alpha}_{i}$ :

$$
b_{i i}(\delta, \mathbf{G}) \bar{\alpha}_{i}>\sum_{j \neq i}\left|b_{i j}(\delta, \mathbf{G})\right| \bar{\alpha}_{j} \quad \text { for all } i \in \mathcal{N} .
$$

Using (19), we find that, when $\delta=0,20$ is satisfied since it boils down to $\bar{\alpha}_{i}>0$. Furthermore, observing that both sides of (20) are continuous functions of $\delta$, we infer that 20) must also be satisfied when $\delta$ belongs to an open interval $(0, \bar{\delta}(\phi, \mathbf{G}))$, where $\bar{\delta}(\phi, \mathbf{G})$ is determined as follows: if there exists a solution (in terms of $\delta$ ) to the equation

$$
\min _{i \in \mathcal{N}}\left(b_{i i}(\delta, \mathbf{G}) \bar{\alpha}_{i}-\sum_{j \neq i}\left|b_{i j}(\delta, \mathbf{G})\right| \bar{\alpha}_{j}\right)=0
$$

within the interval $\left(0,1 / \lambda_{1}(\mathbf{G})\right)$, then $\bar{\delta}(\phi, \mathbf{G})$ is defined as a minimum of such solutions, otherwise we set $\bar{\delta}(\phi, \mathbf{G}) \equiv 1 / \lambda_{1}(\mathbf{G})$.

In general, none of the two conditions (8) and (20) implies the other. To illustrate, set $N=5$ and compare two simple examples: the Salop-type (circular) network and the Chen-Riordan-type (star-shaped) network (Figure 2). For both these networks, we have $\lambda_{1}(\mathbf{G})=2$, hence (8) amounts to the requirement that $\delta<1 / 2$. As for the dominance condition (20), it takes, respectively, the following forms:

$$
\begin{array}{cc}
\text { Salop, } N=5: & \text { Chen-Riordan, } N=5: \\
\delta<\frac{\sqrt{5}-1}{2} \approx 0.618 & \delta<\frac{1+4 \phi}{4\left(1+\phi+3 \phi^{2}\right)}
\end{array}
$$

Figure 3 plots the upper bounds of $\delta$ imposed by, respectively, (8) and (20) for the circle network with $N=5$ nodes (left panel) and for the star-shaped network with $N=5$ nodes (right panel).

As illustrated by Figure 3 , whether condition (8) is stronger or weaker than the diagonal dominance condition (20) depends on the network structure. This is why we assume both these conditions to hold in what follows.

To sum up, there exists a positive threshold value $\bar{\delta}(\phi, \mathbf{G})$ of $\delta$, such that (8) and (20) hold simultaneously for all $\delta<\bar{\delta}(\phi, \mathbf{G})$. This condition will also play an important role for existence and uniqueness of equilibrium (see Proposition 1 in Section 3.4).

\subsection{Firms}

Each firm $i \in \mathcal{N}$ faces the aggregate demand (17) for its variety, and seeks to maximize its profit given by $\pi_{i} \equiv p_{i} X_{i}$. Using 17 , this yields 12

\footnotetext{
${ }^{12}$ For simplicity, we impose the standard assumption that there is no cost of production. Assuming a constant marginal cost $c>0$ will not change any of our results.
} 
Figure 3: Upper bounds of $\delta$ for Chen-Riordan and Salop networks with $N=5$
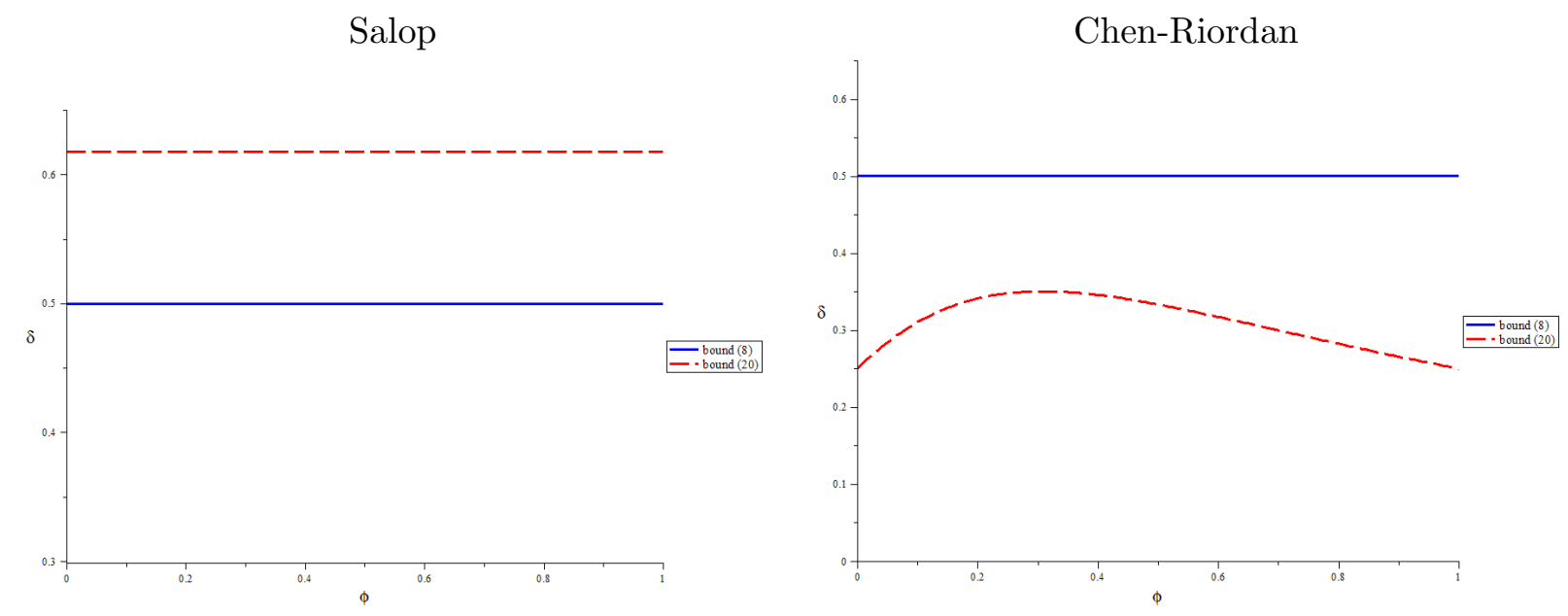

$$
\pi_{i}=\frac{N}{\beta} p_{i}\left(\sum_{j \in \mathcal{N}} b_{i j} \bar{\alpha}_{j}-b_{i i} p_{i}-\sum_{j \neq i} b_{i j} p_{j}\right) .
$$

where $b_{i j}$ are the elements of $\mathbf{B}$ (see (11)). Firm $i$ chooses $p_{i}$ that maximizes (21). The first-order conditions are given by

$$
2 b_{i i} p_{i}+\sum_{j \neq i} b_{i j} p_{j}=\sum_{j \in \mathcal{N}} b_{i j} \bar{\alpha}_{j}
$$

where $i \in \mathcal{N}$.

Since $\pi_{i}$ is strictly concave in $p_{i}$, the second-order conditions hold automatically, given that the first-order condition holds. Solving $(22)$ for $p_{i}$, we obtain the best-reply function of firm $i$ :

$$
p_{i}^{*}\left(\mathbf{p}_{-i}, \boldsymbol{G}\right)=\frac{1}{2}\left(\bar{\alpha}_{i}+\sum_{j \neq i} \frac{b_{i j}}{b_{i i}}\left(\bar{\alpha}_{j}-p_{j}\right)\right) .
$$

An interesting result here is that, except when the network is of Chamberlinian type, the price game does not always exhibit strategic complements. This is to be contrasted with the literature on non-spatial Bertrand competition, where most games display strategic complementarities (see Vives, 1999). Indeed, it is easily verified that

$$
\frac{\partial p_{i}^{*}\left(\mathbf{p}_{-i}, \boldsymbol{G}\right)}{\partial p_{j}}=-\frac{1}{2} \frac{b_{i j}}{b_{i i}}
$$

Observe that, when $(8)$ holds, we have $b_{i i}>0$. However, this is not necessarily true for $b_{i j}{ }^{13}$ More

\footnotetext{
${ }^{13}$ Observe that under Chamberlinian type of network (Figure 2a), the network is complete so that all firms are in direct competition with each other and we are back to the standard case with no network. In that case, if the direct
} 
precisely, as implied by (11), $b_{i j}>0$ if and only if the geodesic distance between $i$ and $j$ is even while the opposite is true when geodesic distance between $i$ and $j$ is odd. In particular, if $g_{i j}=1$, i.e. goods $i$ and $j$ are close substitutes $\left(d_{i j}=1\right)$, then $b_{i j}<0$ and therefore prices $p_{i}$ and $p_{j}$ are

strategic complements. If $g_{i j}=0$ and $g_{i j}^{[2]}=1$, i.e. goods $i$ and $j$ are less close substitutes (distance $2)$, then $b_{i j}>0$ and therefore prices $p_{i}$ and $p_{j}$ are strategic substitutes.

The intuition of this result can be expressed as follows: my enemy's enemy is my friend. This echoes the paper by Arie et al. (2015) who show that, if two small firms serve two separate markets and a large firm serves both these markets, then, under Bertrand competition, a merger is profitable for each of the small firms.

\subsection{Equilibrium}

An equilibrium price vector $\mathbf{p}^{*}$ is a non-negative solution of $(22)$, or, equivalently, a fixed point of the best-reply mapping given by $(23)$. The following proposition shows that a unique equilibrium exists when $\delta<\bar{\delta}(\phi, \mathbf{G})$, where $\bar{\delta}(\phi, \mathbf{G})$ is the threshold value introduced at the end of Section 3.2.

Proposition 1. Assume that $\delta<\bar{\delta}(\phi, G)$, so that (8) and (20) are satisfied simultaneously. Then, there exists a unique interior Nash equilibrium $\left(\mathbf{p}^{*}, \mathbf{X}^{*}\right)$ where $\mathbf{p}^{*}$ is given by

$$
\mathbf{p}^{*}=(\mathbf{I}+\delta \widetilde{\boldsymbol{G}})^{-1} \widetilde{\boldsymbol{\alpha}}(\phi, \boldsymbol{G})
$$

with

$$
\widetilde{\alpha}_{i}(\phi, \boldsymbol{G}) \equiv \frac{\bar{\alpha}_{i}(\phi, \boldsymbol{G})}{1+b_{i i}}
$$

and

$$
\widetilde{g}_{i j} \equiv \frac{b_{j j}}{1+b_{i i}} g_{i j}
$$

and where $\mathbf{X}^{*}$ is given by 17 .

As implied by (25), the equilibrium price vector is proportional to something which has a flavor of a centrality measure from the Bonacich family. Hence, our results bear some resemblance with those obtained by Ballester et al. (2006), but there are at least two major differences. First, in our model the equilibrium price vector (25) is no longer proportional to the Bonacich centrality with a positive discount factor (as it is in Ballester et al., 2006), but to the sign-alternating Bonacich centrality (9). This is because here we are modeling competition (rather than "positive"spillovers, such as education or crime) by means of a network. Second, the network generating the Bonacich centralities in equilibrium depends on $\widetilde{\mathbf{G}}$ instead of $\mathbf{G}$. Clearly, $\widetilde{\mathbf{G}}$ describes a weighted directed network constructed on the basis of the original network $\mathbf{G}$, the weight $\widetilde{g}_{i j}$ of the $i j$-th substitutability link being defined by (27). The intuition behind this "weighting" procedure is easy to grasp: while $g_{i j}$ indicates direct substitutability between $i$ and $j$, the factor $b_{j j} /\left(1+b_{i i}\right)$ captures the relative appeal of variety $i$ compared to $j$, which depends on the positions of varieties $i$ and $j$ in the network. It is also worth noting that the matrix $\widetilde{\mathbf{G}}$ is generically not symmetric, which

competitors of firm $i$ decrease their prices, they will attract more consumers and thus $i$ 's best-reply is to also decrease her price. As a result, prices are strategic complements. 
captures the fact that the relative position of variety $i$ in the network compared to $j$ may be not the same as that of $j$ compared to $i$.

To better understand these results, in the Online Appendix 1, we calculate the equilibrium prices and aggregate demands for the two extreme cases of competition: Chamberlinian competition (complete network in Figure 2a) and Chen-Riordan competition (star network in Figure 2d).

Equation 25) captures radical differences of our model with the standard CES models of monopolistic competition, in which firms set the same profit-maximizing markups regardless of their productivity and/or salience coefficients of their products in the consumer's utility function. Furthermore, the markup in these models depends only on the elasticity of substitution, which is an inverse measure of product differentiation. On the contrary, in our model, prices (which coincide with markups since production is assumed to be costless) not only depend on $\delta$, which indicates the degree of product differentiation, but also on market segmentation, which is captured by the network $\mathbf{G}$, and on the spatial discount factor $\phi$, which captures the role of proximity effect.

\subsection{The case when $\beta \rightarrow 0$}

The above results have been obtained for the case when $\beta$ is strictly positive, i.e. when consumers love variety and consume a positive quantity of all goods. This makes our modeling setup closer to the non-spatial setting a la Chamberlain-Dixit-Stiglitz rather than the spatial models proposed by Hotelling and Salop. We now show that things change dramatically when $\beta \rightarrow 0$, in which case our model has much more in common with the standard spatial models.

When we set $\beta=0$ in (3), the utility function becomes linear. In this case, consumer $k$ buys at most one differentiated variety. Indeed, if

$$
\max _{i \in \mathcal{N}} \frac{\alpha \phi^{d_{i k}}}{p_{i}} \geq 1,
$$

consumer $k$ spends her whole income on variety $i^{*}(k)$, which is given by

$$
i^{*}(k) \equiv \arg \max _{i \in \mathcal{N}} \frac{\alpha \phi^{d_{i k}}}{p_{i}} .
$$

Otherwise, consumer $k$ does not buy any good since she spends her whole income on the outside good. Consumer $k$ 's individual demand for variety $i$ is then given by:

$$
x_{i k}= \begin{cases}\frac{Y_{k}}{p_{i}}, & \text { when } i=i^{*}(k), \\ 0, & \text { otherwise. }\end{cases}
$$

Therefore, the market demand for variety $i$ takes the form:

$$
X_{i}=\frac{1}{p_{i}} \sum_{k \in \mathcal{N}} Y_{k} \cdot \mathbb{I}\left\{i=i^{*}(k)\right\},
$$

where $\mathbb{I}_{S}$ stands for the indicator of a set $S$.

This demand system is in the spirit of Sattinger (1984) and Perlof and Salop (1985), which may be viewed as a probabilistic-choice-based counterpart of the Hotelling's (1929) and Salop's (1979) 
spatial settings. Since production is assumed to be costless, the profit function of firm $i$ is given by

$$
\pi_{i} \equiv p_{i} X_{i}=\sum_{k \in \mathcal{N}} Y_{k} \cdot \mathbb{I}\left\{i=i^{*}(k)\right\} .
$$

As a consequence, the profit function is a non-increasing function in $p_{i}$, and no equilibrium with positive prices exists, since there will always be an incentive for firm $i$ to undercut a competitor who has a positive market share. Hence, the model boils down to standard Bertrand competition.

\section{Comparative statics}

Let us now examine how the different parameters of the model affect the equilibrium prices. We will consider the impact of variation in the degree of $\delta$ substitutability and the spatial discount factor $\phi$ on equilibrium prices.

\subsection{A change in $\delta$}

How prices $\mathbf{p}^{*}$ and aggregate demand $\mathbf{X}^{*}$ vary with $\delta$, which is the inverse measure of product differentiation, looks pretty complicated and is general ambiguous.

\subsubsection{Analytical results}

Proposition 2. There exists a positive threshold value $\widehat{\delta}(\phi, \mathbf{G})$ of $\delta$, such that, when $\delta<\widehat{\delta}(\phi, \mathbf{G})$, we have $\partial \mathbf{p}^{*} / \partial \delta<\mathbf{0}$, i.e. an increase in toughness of local competition $\delta$ leads to lower prices.

The intuition behind Proposition 2 is as follows: when products are highly locally differentiated, a small reduction in the degree of differentiation makes competition tougher and reduces all prices. However, the magnitude of price reduction depends on both the network structure and the distance decay factor. We illustrate this below by means of numerical simulations.

\subsubsection{Numerical simulations}

How do changes in $\delta$ affect equilibrium prices when $\delta>\widehat{\delta}(\phi, G)$ ? Since providing analytical results for this case is problematic, we resort to numerical simulations.

To illustrate how the equilibrium prices vary with $\delta$, consider a star-shaped network (ChenRiordan competition) with $N=6$. Does the firm located in the center always enjoy higher monopoly power than the other firms? The answer is no. The firm located in the center has better access to the market than the peripheral firms, but it also faces tougher competition (five direct competitors instead of one). Which of the two effects prevails depends on the value of the substitutability parameter $\delta$. Figure 4 reports the prices of the central firm (the star) and a peripheral firm, obtained by means of a simulation where $\phi=0.6, \alpha=1$, while $\delta$ varies between 0 and 0.2 , the step of the grid being 0.01 .

As shown by Figure 4, the central firm charges a higher price than the peripheral firms if and only if $\delta$ does not exceed a threshold value, which is approximately 0.15 . When $\delta$ is above 0.15 , 
Figure 4: Changes in equilibrium prices in the Chen-Riordan network for $N=6$ and $\phi=0.6$

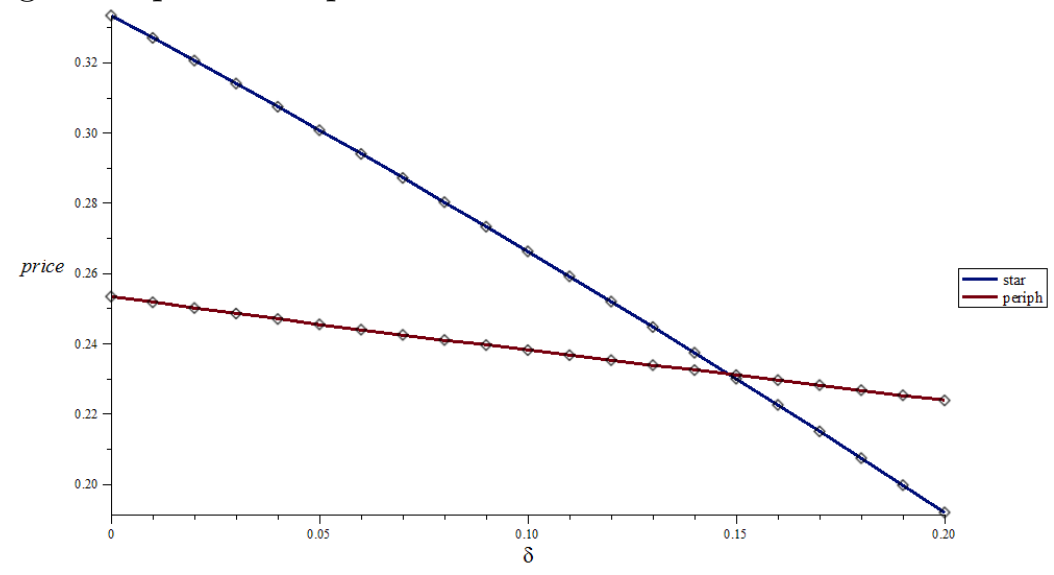

the competition effect deprives the central firm of so much monopoly power that it starts charging a lower price compared to the peripheral firms. We also performed simulations for star-shaped networks with a number of nodes different from 6 . The results are qualitatively the same as in Figure 4.

In the Online Appendix 2.1, we perform the same exercise for the line or chain network (Hotelling competition) with $N=5$. The results are displayed in Figure A1 and they are similar to that of Figure 4.

Another question of interest is how the bound $\widehat{\delta}(\phi, \mathbf{G})$ varies with $\phi$. To illustrate this, consider a star-shaped network with $N=10$ firms. In this case, we have $\lambda_{1}(\mathbf{G})=3$, hence the invertibility condition (8) takes the form of $\delta<1 / 3$. As for the diagonal dominance condition (20), it is readily verified that this condition becomes:

$$
\delta<\frac{1+9 \phi}{9\left(8 \phi^{2}+\phi+1\right)}
$$

For this special case, comparative statics of the price equilibrium with respect to $\delta$ is illustrated by Figure 5, which depicts the admissible domain of the two key parameters of the model: $\phi$ and $\delta$. In the horizontally hatched area, both (8) and (20) hold (meaning that a unique interior price equilibrium exists, as implied by Proposition 1), and all prices decrease in response to an increase in $\delta$. The upper boundary of this area is the plot of the bound $\bar{\delta}(\phi, \mathbf{G})$ from Proposition 1 as a function of $\phi$. In the area with diagonal hatching, the diagonal dominance condition (20) is no longer satisfied, but a unique interior price equilibrium still exists, and all prices still decrease as $\delta$ increases. In the two white areas, although a unique interior price equilirium still exists, the price charged by periphery firms start increasing with $\delta$, while the price charged by the star firm continues to decrease. Finally, in the shaded area, no interior price equilibrium exists, even though condition (8) still holds. Thus, when $\mathbf{g}$ is a star-shaped network with $N=10$ firms, the plot of $\widehat{\delta}(\phi, \mathbf{G})$ as a function of $\phi$ is given by the upper boundary of the area with diagonal hatching. Note that we have $\widehat{\delta}(\phi, \mathbf{G})>\bar{\delta}(\phi, \mathbf{G})$, i.e. the conditions of Proposition 2 are less stringent than those 
Figure 5: Comparative statics of prices in $\delta$ in the Chen-Riordan network for $N=10$

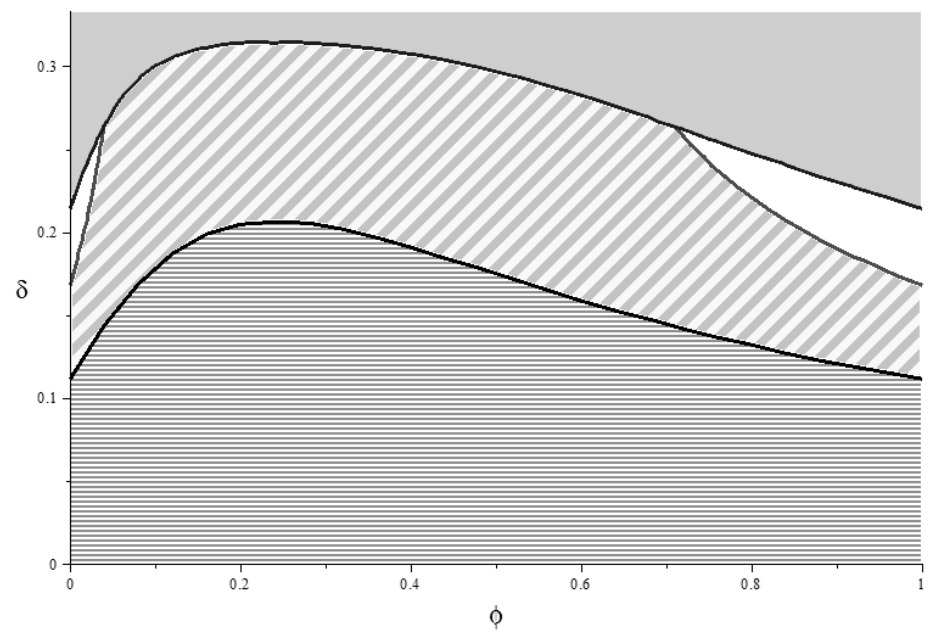

of Proposition 1, 14

The intuition why prices can increase with $\delta$ is as follows. An increase in $\delta$ makes competition tougher, hence, given that all other prices remain the same, star firm's best reply is to reduce its price. This is a direct effect. There is, however, an indirect effect, which exists because the prices of periphery firms also change. On the one hand, a reduction in the star firm's price shifts the prices of periphery firms downwards, as the prices of neighboring firms are strategic complements. On the other hand, prices charged by any two periphery firms $i$ and $j$ are strategic substitutes to each other. As a consequence, $p_{j}$ should increase in response to a drop in $p_{i}$, and vice versa. This latter effect tends to dominate both the direct effect of $\delta$ and the strategic complementarity effect as there are sufficiently many periphery firms.

\subsection{A change in $\phi$}

We now turn to studying how the equilibrium $\mathbf{p}^{*}$ varies with the spatial discount factor $\phi$. Just like in the previous section, we are able to state clear-cut analytical results for the values of $\delta$ which do not exceed a certain threshold level, while otherwise we proceed with simulations.

\subsubsection{Analytical results}

We have the following proposition:

Proposition 3. There exists a positive threshold value $\widetilde{\delta}(\phi, \mathbf{G})$ of $\delta$, such that, when $\delta<\widetilde{\delta}(\phi, \mathbf{G})$, we have $\partial \mathbf{p}^{*} / \partial \phi>\mathbf{0}$, i.e. an increase in spatial discount factor $\phi$ leads to higher prices.

The intuition behind this result is as follows: when $\phi$ increases, firm $i$ will have less monopoly power over consumer $i$, but better access to all the other consumers. The latter effect clearly

\footnotetext{
${ }^{14}$ Although not proven anaytically, this seems to be true in general. After running a long series of simulations with networks of different sizes and configurations, we were unable to find a single example where $\widehat{\delta}(\phi, \mathbf{G})>\bar{\delta}(\phi, \mathbf{G})$ fails to hold.
} 
dominates the former when $\delta<\widetilde{\delta}(\phi, \mathbf{G})$. This result stresses the difference between our approach and spatial competition models a la Hotelling. In these models, a reduction in transport costs which is a natural counterpart to our exercise of increasing $\phi$ - lead to tougher competition, whence lower prices. This is presumably because spatial competition models rest on the discrete choice premise, while in our model it is the love-for-variety effect that boosts the market access effect.

\subsubsection{Numerical simulations}

Let us now understand how $\phi$ affects equilibrium prices when products are only slightly differentiated, i.e. when $\delta>\widetilde{\delta}(\phi, \mathbf{G})$. Consider a star-shaped network (Chen-Riordan competition) with $N=6$. We look at how the equilibrium prices charged by firms located, respectively, at the "star" node and at the "peripheral" nodes vary with the spatial discount factor $\phi$. Figure 4 displays the results when $\delta=0.15$ (which means relatively low substitutability across neighboring varieties, i.e. softer local competition).

Figure 6: Changes in equilibrium prices in the Chen-Riordan network for $N=6$ and $\delta=0.15$

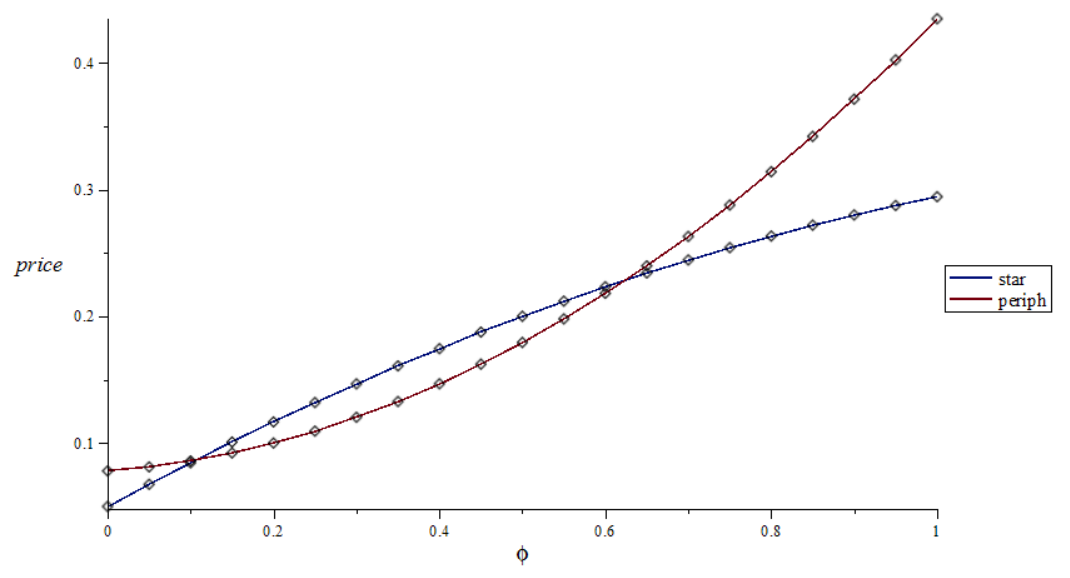

When $\delta=0.15$, we find that the "star" firm enjoys higher monopoly power (that is, charges higher price) than the "peripheral" firms if $\phi$ lies in an intermediate domain (from 0.1 to 0.625 ). Otherwise, peripheral firms price at a higher level. This may be explained by a considerable advantage the "star" firm gains from being a "star" in having better access to the markets. This advantage fades when $\phi$ is close to 0 (which means little access to markets other than the local market of a firm) or to 1 (all firms have almost complete access to all markets). Indeed, on the one hand, being a "star" does not yield better market access than the others have. On the other hand, the "star" competes directly with everyone (whereas a "peripheral" firm competes directly with the "star" only), thus bearing a burden of high competitive pressure. As a result, when $\phi$ is close to either 0 or 1 , the price of the "star" is lower.

In the Online Appendix 2.2, we perform the same exercise when $\delta=0.3$. The results are displayed in Figure A2. We show that being a "star" is even less of an advantage. In the Online Appendix 2.2, we also perform the same exercise but for the line or chain network (Hotelling competition) with $N=5$ and for $\delta=0.15$. The results are displayed in Figure A3. We find results 
Figure 7: Comparative statics of prices in $\phi$ in the Chen-Riordan network for $N=10$

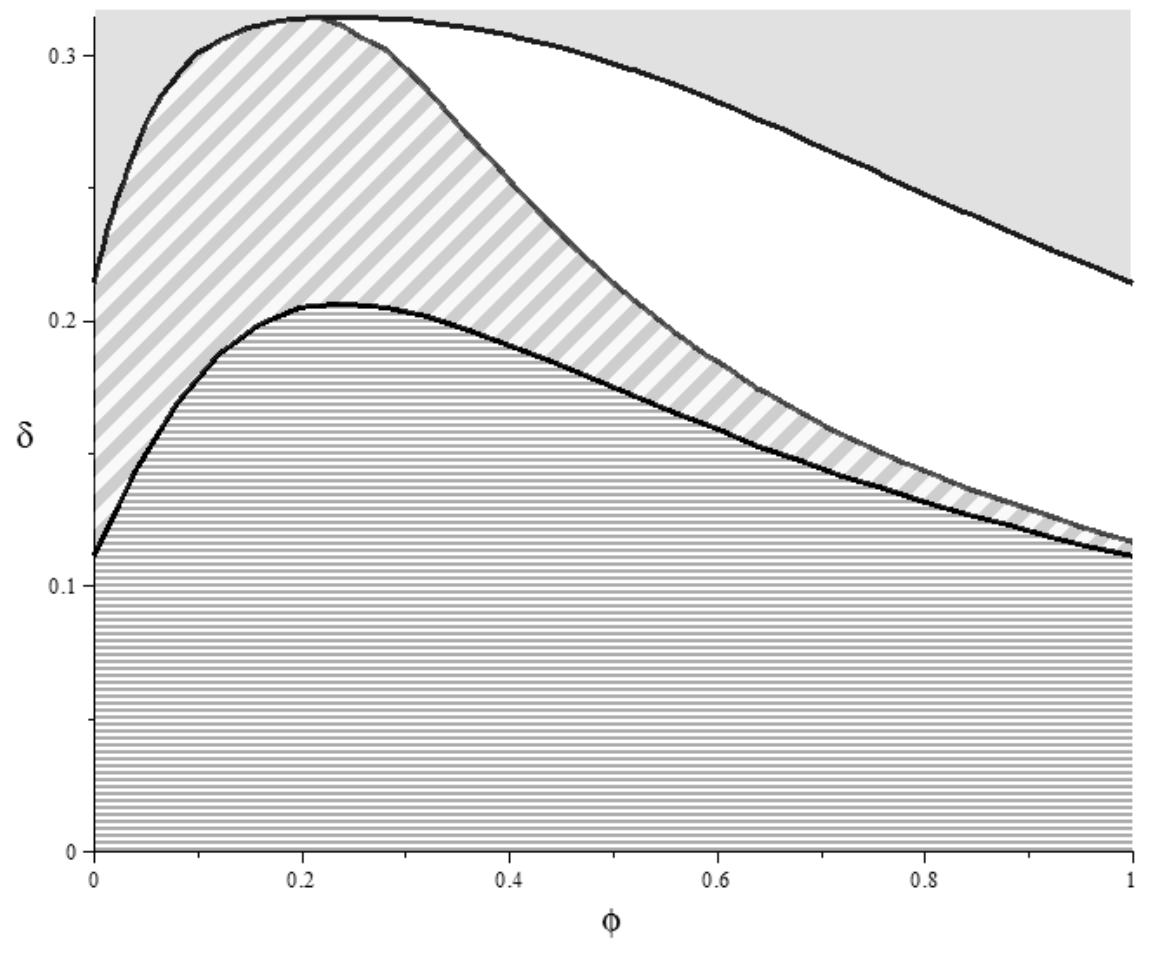

similar to that of Figure 6 .

Finally, to see how the bound $\widetilde{\delta}(\phi, \mathbf{G})$ varies with $\phi$, consider again the example of a ChenRiordan-type network with $N=10$ firms. In this case, the variation of prices with respect to $\phi$ is illustrated by Figure 7 .

As in Figure 5, the horizontally hatched area in Figure 7 is when $\delta<\bar{\delta}(\phi, \mathbf{G})$, i.e. when conditions (8) and 20 both hold. The upper boundary of this area plots the bound $\bar{\delta}(\phi, \mathbf{G})$ as a function of $\phi$. In this area, all prices increase in $\phi$. The area with diagonal hatching is where condition (20) does not hold anymore, but a unique interior price equilibrium still exists, and all prices still increase in $\phi$. The upper boundary of this area plots the bound $\widetilde{\delta}(\phi, \mathbf{G})$ as a function of $\phi$. In the white area, a unique interior price equilibrium still exists, but the price charged by the star firm decreases with $\phi$, although prices charged by perophery firms decrease with $\phi$. Finally, in the shaded area, i.e. when $\delta$ is high enough, an interior price equilibrium no longer exists.

The intuition of a possible reduction in price charged by the star firm under an increase in $\phi$ is as follows. A higher $\phi$ means that each consumer has a higher willingness to pay for all varieties other than her ideal variety. Using the terminology of the international trade literature, a higher $\phi$ mitigates the home bias effect. As a result, each firm has better access to the market but competition becomes tougher. Clearly, as the star-shaped firm competes directly with all the periphery firms, it faces tough competition. This is why, when $\phi$ increases, the star firm starts losing the advantage due to its central location, as each location $i$ becomes more accessible for a periphery firm $j \neq i$. Consequently, the market access effect for the star firm becomes weaker and weaker and, at some point, the competition effect becomes the dominant force. Note that, as seen 
in Figure 7, the higher $\phi$, the broader the range of the $\delta$ values under which the competition effect dominates the market access effect for the star firm (i.e. the white area becomes broader as $\phi$ increases).

\subsection{Network versus non-network effects}

What do we gain by having a network approach to modeling markets of differentiated goods? To shed light on this question, we illustrate here the difference between "network" and "non-network" effects. Assume $\phi=1$. Then, in the complete network (Chamberlinian competition), we have pure monopolistic competition since

$$
p^{*}=\frac{\alpha(1-\gamma)}{2(1-\gamma)+\gamma(n-1)}
$$

This is exactly the result for the benchmark monopolistic competition model without network (see Combes et al., 2008, Chap. 3). If we differentiate (28), we obtain:

$$
\frac{\partial p^{*}}{\partial \alpha}>0, \frac{\partial p^{*}}{\partial n}<0 \text { and } \frac{\partial p^{*}}{\partial \gamma} \gtreqless 0 \Leftrightarrow \gamma n \lesseqgtr 1 .
$$

As shown by the above results, with a more general approach in terms of networks, the comparativestatics results are quite different. For example, we have shown the importance of the spatial discount factor $\phi$ in evaluating the impact of substitutability $\delta$ on the market prices. Also, we have seen that these comparative-statics results crucially depend on the network structure and that the most "central" firms in a network do not always enjoy higher monopoly power than other firms and thus do not always charge higher prices. We have seen, for example, that for a star-shaped network, the firm located in the star node (the most "central" firm) does not always enjoy higher monopoly power than the other firms. This is because the firm located at the star node has better access to the market than the peripheral firms, but it also faces tougher competition (all peripheral firms instead of one).

\section{Symmetric equilibrium with regular networks}

In the above section, we have studied how the market outcome varies with the degree of substitutability $\delta$ and the distance decay factor $\phi$. Another question of interest is how the equilibrium responds to changes in the structure of the network. For example, what happens when competition gets less localized, i.e. when the network is denser so that new links emerge without breaking the old ones? There is no straightforward answer in general networks. To obtain clear-cut results, we now consider a special case when the equilibrium in the price-setting game is symmetric, i.e. when $p_{i}^{*}=p^{*}$ for all $i \in \mathcal{N}$.

As implied by (23), the necessary and sufficient condition for the equilibrium to be symmetric is given by:

$$
\bar{\alpha}_{i}(\phi, \boldsymbol{G}) \simeq \sum_{j \in \mathcal{N}} g_{i j} b_{j j}
$$


where $\backsim$ denotes proportionality. The right-hand side of $(29)$ is independent of $\phi$, while the lefthand side is, by (16), a polynomial of $\phi$ but is also independent of $\delta$. Hence, (29) holds for any admissible values of $\phi$ and $\delta$ if and only if both $\bar{\alpha}_{i}(\phi, \boldsymbol{G})$ and $b_{i i}$ are the same for all $i \in \mathcal{N}$. Furthermore, $\bar{\alpha}_{i}(\phi, \boldsymbol{G})=\bar{\alpha}_{j}(\phi, \boldsymbol{G})$ is equivalent to the following condition:

$$
\text { for any } k \leq d \text {, and for any } i, j \in \mathcal{N}, N_{k}(i)=N_{k}(j) \text {. }
$$

Condition (30) tells us that, for a symmetric equilibrium to exist, the network $(\mathcal{N}, \mathbf{G})$ has to be "symmetric" in some sense. The weakest concept of symmetry for networks is regularity. Recall that a network is regular if all nodes have the same degree $r<N$, which is known as the valency of a regular graph. However, regularity of $(\mathcal{N}, \mathbf{G})$ is a necessary, but not sufficient condition for (30) to hold. A counterexample is given by the Frucht graph (Biggs, 1974, Chap. 15). See the Online Appendix 3 for details and Figure A4 for a representation of the Frucht graph.

A sufficient condition for (30) is that the network is vertex transitive. Vertex transitivity means that, for any $i, j \in \mathcal{N}$, there exists an automorphism of $(\mathcal{N}, \mathbf{G})$ that maps $i$ into $j$. Moreover, vertex transitivity implies that $b_{i i}=b_{j j}$ for all $i \in \mathcal{N}$ also holds. More intuitively, the network is vertex transitive if all nodes have the same centrality measure (this has to be true for any centrality measure) ${ }^{15}$ Clearly, both the complete (Chamberlain-type) network and the circular (Salop-type) network are vertex transitive.

Observe that a network is regular if and only if $r^{-1} \mathbf{G}$ is a bistochastic matrix. In this case, we have $r=\lambda_{\max }(\mathbf{G})$. Thus, (8) boils down to $\gamma r<1$. The unique interior symmetric equilibrium price $p^{*}$ is then given by:

$$
p^{*}=\frac{\alpha}{N} \frac{1+\phi r+\phi^{2} N_{2}+\ldots+\phi^{d} N_{d}}{1+(1+\delta r) b},
$$

where $b \equiv b_{i i}=1+\delta^{2} r-\delta^{3} g_{i i}^{[3]}+\ldots$

Using equation (31), we can study how prices change when competition becomes less localized, which we model by adding new links to the network without removing the existing ones, and keeping the set of nodes unchanged. To be precise, assume that the network changes from $(\mathcal{N}, \mathbf{G})$ to $\left(\mathcal{N}, \mathbf{G}^{\prime}\right)$, where $(i)$ both $\mathbf{G}$ and $\mathbf{G}^{\prime}$ are regular, and $(i i) \mathbf{G}^{\prime}$ is obtained from $\mathbf{G}$ by adding new links, without removing the old ones. In particular, this means that the valency $r^{\prime}$ of $\mathbf{G}^{\prime}$ exceeds $r$, which is the valency of $\mathbf{G}$. We have the following result:

Lemma 2. Assume that $\delta$ is not too large while $\phi \in(0,1)$. Then, both the numerator and the denominator of (31) increase when $\mathbf{G}$ changes to $\mathbf{G}^{\prime}$.

The intuition behind this lemma is as follows. When competition gets less localized, two effects are at work. First, the numerator of (31) captures the market access effect, which results in skewing the distribution of distances (how many nodes are at distance 1 from $i$, at distance 2 from $i$, etc.) toward zero, thus bringing all consumers closer to each firm. Second, the numerator of (31) captures the competition effect since competition gets tougher as new links arise. The lemma basically states

\footnotetext{
${ }^{15}$ For example, the Frucht graph is regular but nodes do not have the same eigenvector centrality, which entails asymmetric distance distribution.
} 
that the two effects work in the opposite directions: the market access effect always drives prices upwards, while competition effect leads to a reduction in prices. Which of the two effects dominates is a priori ambiguous. Indeed, the total change in prices is given by

$$
\Delta p^{*}=\frac{\alpha}{N}\left[\frac{1+\phi r+\phi^{2} N_{2}+\ldots+\phi^{d} N_{d}+\mathrm{MA}(\phi)}{1+(1+\gamma r) b+\mathcal{O}(\delta)}-\frac{1+\phi r+\phi^{2} N_{2}+\ldots+\phi^{d} N_{d}}{1+(1+\gamma r) b}\right]
$$

where

$$
\mathrm{MA}(\phi) \equiv \phi \Delta r+\phi^{2} \Delta N_{2}+\ldots+\phi^{d} \Delta N_{d}
$$

and

$$
\operatorname{Comp}(\delta) \equiv\left(\delta+\delta^{2}\right) \Delta r+\mathcal{O}\left(\gamma^{3}\right) .
$$

The intuition behind the terms $\operatorname{MA}(\phi)$ and $\operatorname{Comp}(\delta)$ is easy to grasp. The term $\operatorname{MA}(\phi)$ captures the market access effect, which is the higher the more skewed the distance distribution gets to the left when new substitutability links emerge. In other words, $\operatorname{MA}(\phi)$ is a measure of a change in firms' closeness to the whole population of consumers. It is worth noting that $\mathrm{MA}(\phi)$ varies with the spatial discount factor $\phi$, but not with the substitutability $\delta$. On the contrary, the term $\operatorname{Comp}(\delta)$ depends on $\delta$, but not on $\phi$. This term captures the competition effect stemming from better substitution across varieties due to a denser network. To sum up, introducing $\operatorname{MA}(\phi)$ and $\operatorname{Comp}(\delta)$ provides a clear comparison of the roles played by the parameters $\phi$ and $\delta$ in our model: the former governs the market-access effect, while the latter shows the intensity of competition effect.

Clearly, $\Delta p^{*}>0$ if and only if

$$
\frac{\operatorname{MA}(\phi)}{\operatorname{Comp}(\delta)}>\frac{N}{\alpha} p^{*}
$$

i.e. when the market size effect dominates over the competition effect. Whether this condition holds is a priori unclear. The following proposition gives an answer.

Proposition 4. Assume that $(i)$ the product variety network is regular, and $(i i) \delta$ is not too high. Then, there exist two threshold values, $\phi, \bar{\phi} \in(0,1)$ of the distance decay factor $\phi$ such that, when the network becomes denser, equilibrium prices decrease if and only if $\phi<\phi<\bar{\phi}$. Otherwise, the market access effect dominates the competition effect and equilibrium prices increase when the network becomes denser.

This result concurs with that of Arie et al. (2015) who study the impact of multimarket contacts between firms on the toughness of competition. Although these authors work with a very different setting from ours, they find a non-monotonic relationship between firms' prices and markets shares. We differ from them by capturing a varying degree of overlap between markets served by different firms (closer firms compete more fiercely) while, in Arie et al. (2015), each market is either private or fully overlapping. 


\section{$6 \quad$ Welfare analysis}

We now study the welfare properties of our model. In order to derive aggregate welfare, we first compute the aggregate consumer surplus. Inverting the market demands (15) yields:

$$
\mathbf{p}=\overline{\boldsymbol{\alpha}}(\phi, \boldsymbol{G})-\frac{\beta}{N}(\mathbf{I}+\delta \boldsymbol{G}) \mathbf{X}
$$

Such an inverse demand system can be generated by an aggregate representative consumer whose preferences are given by

$$
\mathcal{U}(\mathbf{X})=X_{0}+\overline{\boldsymbol{\alpha}}^{T} \mathbf{X}-\frac{\beta}{2 N} \mathbf{X}^{T}(\mathbf{I}+\delta \boldsymbol{G}) \mathbf{X} .
$$

Aggregate consumer surplus is standardly defined by $C S \equiv \mathcal{U}(\mathbf{X})-\mathbf{p}^{T} \mathbf{X}-X_{0}$ and is given by:

$$
C S=\frac{N}{2 \beta}(\overline{\boldsymbol{\alpha}}-\mathbf{p})^{T} \mathbf{B}(\overline{\boldsymbol{\alpha}}-\mathbf{p}) .
$$

The next step is to compute the aggregate profit $\Pi \equiv \sum_{i \in \mathcal{N}} \pi_{i}=\mathbf{p}^{T} \mathbf{X}$. Using (17), we obtain:

$$
\Pi=\frac{N}{\beta} \mathbf{p}^{T} \mathbf{B}(\overline{\boldsymbol{\alpha}}-\mathbf{p}) .
$$

Hence, the social welfare, defined as $\mathbb{W} \equiv C S+\Pi$, is given by:

$$
\mathbb{W}=\frac{N}{2 \beta}\left(\overline{\boldsymbol{\alpha}}^{T} \mathbf{B} \overline{\boldsymbol{\alpha}}-\mathbf{p}^{T} \mathbf{B} \mathbf{p}\right)
$$

Because $\mathbf{B}$ is a positive-definite matrix, it follows immediately from 32 that the social welfare is maximized when prices are competitive, i.e. when $\mathbf{p}=\mathbf{0}$. This is the first best and, because of externalities created by the network, it cannot be achieved in equilibrium where prices are determined by (25) and depend on the consumers' position in the network.

Let us now determine the equilibrium welfare by plugging the equilibrium prices into (32). First, writing 22 in vector-matrix form leads to:

$$
(\mathbf{D}+\mathbf{B}) \mathbf{p}=\mathbf{B} \overline{\boldsymbol{\alpha}},
$$

where $\mathbf{D} \equiv \operatorname{diag}\left\{b_{11}, \ldots, b_{n n}\right\}$. Solving $(33)$ for prices yields

$$
\mathbf{p}^{*}=(\mathbf{I}+\mathbf{D}+\delta \mathbf{G D})^{-1} \overline{\boldsymbol{\alpha}} .
$$

To obtain the equilibrium welfare level $\mathbb{W}^{*}$, we plug the equilibrium prices (34) into the social welfare defined in (32). We then obtain an explicit expression for $\mathbb{W}^{*}$.

We now study how the equilibrium welfare level $\mathbb{W}^{*}$ varies in response to changes in the productvariety network. To demonstrate several contrasting welfare effects, we focus here on one specific type of shocks: what happens when a network becomes denser, i.e. when new links are added (as in Proposition 4). The effect is unclear ${ }^{16}$ since, in denser product-variety networks, firms compete

\footnotetext{
${ }^{16}$ In games on networks, usually, denser networks lead to higher total welfare (see, e.g. Ballester et al., 2006).
} 
more fiercely and hence they set lower prices, which increases the consumer surplus but reduces firms' profits. Also, when networks become denser, consumers enjoy better proximity to the whole range of varieties. It turns out that the impact of a denser network on equilibrium welfare $\mathbb{W}^{*}$ depends on the spatial discount factor $\phi$. To show this, we run some numerical simulations.

Set $N=6$ and $\delta=0.15$. Let us compare the complete network a la Chamberlin (Figure 2a) and the the chain network a la Hotelling (Figure 2b) since the former is much denser (density equal to 1 ) than the latter (density equal to $2 / n$ ). Figure 5 displays the results.

Figure 8: Chamberlin versus Hotelling: Welfare effects when $N=6$ and $\delta=0.15$

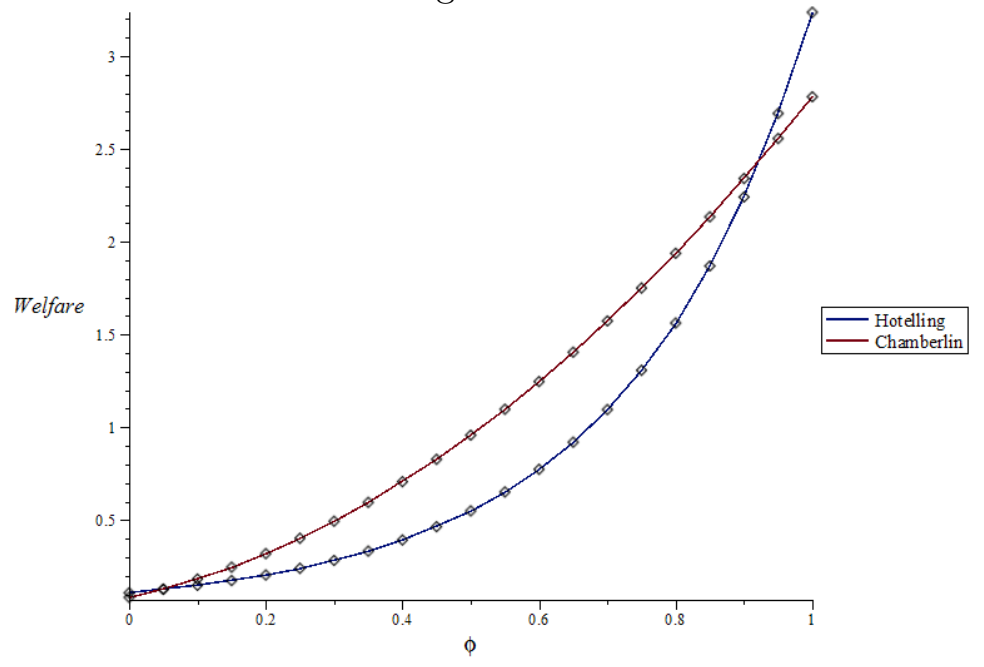

We can see that, when the spatial discount factor $\phi$ is below some threshold level (which is between 0.8 and 0.9 ), adding more links is beneficial to the economy from a welfare perspective. However, when $\phi$ exceeds this threshold and becomes sufficiently close to one, i.e. when proximity of all consumers to all varieties is very high, a denser network leads to welfare losses. Indeed, when $\phi$ takes a very high value, there is considerable reduction of the producer's surplus, which is due to extremely tough Chamberlinian competition, that cannot be fully compensated by a moderate increase in consumer's surplus, which stems from slightly better proximity to the whole range of varieties.

In the Online Appendix 4, we perform the same exercise for two other networks: the circular network a la Salop (Figure 2c) and the star-shaped network a la Chen-Riordan (Figure 2d) and compare them to the complete network a la Chamberlin. Figures A5 and A6 display the results and we find the same qualitative results as in Figure 5. Thus, to sum up, a denser network may result in a lower social welfare level when the spatial discount factor $\phi$ is sufficiently large.

\section{Conclusion}

In this paper, we develop a new model of price competition, which combines features of both spatial and monopolistic competition, thus encompassing two very different aspects of product differentiation: love for variety and consumers' location-specific taste heterogeneity. As a consequence, our 
model allows for a rich set of regimes of imperfect competition. Our idea is to model the product space as a network, where a link between two varieties exists if and only if they are direct substitutes, while consumer's willingness to pay decays with the geodesic distance of a specific variety from her ideal variety. Thus, consumers exhibit love for variety, as in monopolistic competition, but are willing to pay less for more distant varieties, like in the address approach. Chamberlin-type or Hotelling-type spatial structures are obtained as a limiting case when the substitutability network is a complete graph or a chain. We show that there exists a unique Nash equilibrium in prices where each equilibrium price is a function of the sign-alternating Bonacich centrality of the firm. We also investigate how the degree of product differentiation and the spatial discount factor affect the equilibrium prices. We find that, when products are highly differentiated, a small reduction in the degree of differentiation makes competition tougher and reduces all prices. However, the magnitude of price reduction depends on both the network structure and the distance decay factor. If, for example, we consider a star-shaped network, we find that the firm located at the center does not always enjoy higher monopoly power than the other firms. This is because the central firm has better access to the market than the peripheral firms, but it also faces tougher competition. We also study symmetric equilibria and show how denser networks affect prices. Finally, we analyze welfare implications of our model by studying the welfare consequences of a denser network.

We believe that this paper provides a methodological contribution by modeling firms' and consumers' heterogeneity by their position in the network of product varieties. We also believe that our model could be used to analyze issues related to economic geography, international trade, economic growth, etc. We finally hope that our contribution will spur further research in these directions.

\section{References}

[1] Amiti, M., Itskhoki, O.and J. Konings (2016), "International shocks and domestic prices: How large are strategic complementarities?" NBER Working Paper No. 22119.

[2] Anderson, S.P., de Palma, A. and J.-F. Thisse (1992), Discrete Choice Theory of Product Differentiation, Cambridge, MA: MIT Press.

[3] Anderson, S.P., De Palma, A. and Y. Nesterov (1995), "Oligopolistic competition and the optimal provision of products," Econometrica 63, 1281-1301.

[4] Arie, G., Markovich, S. and M. Varela (2015), "The competitive effect of multimarket contact," Simon Business School Working Paper No. FR 12-12, University of Rochester.

[5] Ballester, C., Calvó-Armengol, A. and Y. Zenou (2006), "Who's who in networks: wanted the key player," Econometrica 74, 1403-1417.

[6] Banerji, A. and B. Dutta (2009), "Local network externalities and market segmentation," International Journal of Industrial Organization 27, 605-614. 
[7] Behrens, K. and Y. Murata (2007), "General equilibrium models of monopolistic competition: A new approach," Journal of Economic Theory 136, 776-787.

[8] Behrens, K., Lamorgese, A. R., Ottaviano, G.I.P. and T. Tabuchi (2007), "Changes in transport and non-transport costs: Local vs global impacts in a spatial network," Regional Science and Urban Economics 37, 625-648.

[9] Belleflamme, P. and M. Peitz (2010), Markets and Strategies, Cambridge: Cambridge University Press.

[10] Billand, P., Bravard C., Chakrabarti, S. and S. Sarangi (2014), "A note on networks of collaboration in multi-market oligopolies," Annals of Regional Science 53, 325-335.

[11] Biggs, N.L. (1974), Algebraic Graph Theory, Cambridge: Cambridge University Press.

[12] Bimpikis, K., Ehsani, S. and R. Ilkilic (2015), "Cournot competition in networked markets,"Unpublished paper, Stanford University .

[13] Bloch, F. and N. Quérou (2013), "Pricing in social networks," Games and Economic Behavior 80, 243-261.

[14] Bonacich P. (1987), "Power and centrality: A family of measures," American Journal of Sociology 92, 1170-1182.

[15] Bramoullé, Y., Kranton, R. and M. d'Amours (2014), "Strategic interaction and networks," American Economic Review 104, 898-930.

[16] Bramoullé, Y., Rogers, B.W. and A. Galeotti (2016), The Oxford Handbook of the Economics of Networks, Oxford: Oxford University Press.

[17] Calvó-Armengol, A., Patacchini, E. and Y. Zenou (2009), "Peer effects and social networks in education," Review of Economic Studies 76, 1239-1267.

[18] Camerer, C., Loewenstein, G., and D. Prelec (2005), "Neuroeconomics: How neuroscience can inform economics," Journal of economic Literature 43, 9-64.

[19] Candogan, O, Bimpikis, K. and A. Ozdaglar (2012), "Optimal pricing in networks with externalities," Operations Research 60, 883-905.

[20] Carroni, E. and S. Righi (2015), "Pricing in social networks under limited information," University of Namur, Department of Economics Working Papers No. 2015/03.

[21] Chamberlin, E.H. (1933), The Theory of Monopolistic Competition, Cambridge, MA: Harvard University Press.

[22] Chen, Y. and M.H. Riordan (2007), "Price and Variety in the Spokes Model", Economic Journal 117, 897-921. 
[23] Chen, Y. and M.H. Riordan (2008), "Price-increasing competition," Rand Journal of Economics 39, 1042-1058.

[24] Chen, Y.-J., Zenou, Y. and Zhou, J. (2018), "Competitive pricing strategies in social networks," RAND Journal of Economics, forthcoming.

[25] Combes, P.-P., Mayer, T. and J.-F. Thisse (2008), Economic Geography: The Integration of Regions and Nations, Princeton: Princeton University Press.

[26] Currarini, S. and F. Feri (2015), "Information sharing networks in linear quadratic games," International Journal of Game Theory 44, 701-732.

[27] d'Aspremont, C. and R. Dos Santos Ferreira (2009), "Price-quantity competition with varying toughness," Games and Economic Behavior 65, 62-82.

[28] Deneckere, R.J. and M. Rothschild (1992), "Monopolistic competition and preference diversity," Review of Economic Studies 59, 361-373.

[29] Deroian, F. and F. Gannon (2006), "Quality-improving alliances in differentiated oligopoly," International Journal of Industrial Organization 24, 629-637.

[30] Dhingra, S. (2013), "Trading away wide brands for cheap brands," American Economic Review 103, 2554-2584.

[31] Di Comite, F., Thisse, J. F., and H. Vandenbussche (2014), "Verti-zontal differentiation in export markets," Journal of International Economics 93, 50-66.

[32] Dickson, P.R. and J.L. Ginter (1987), "Market segmentation, product differentiation, and marketing strategy," Journal of Marketing 51, 1-10.

[33] Dixit, A.K. and J.E. Stiglitz (1977), "Monopolistic competition and optimum product diversity," American Economic Review 67, 297-308.

[34] Einav, L. and J. Levin (2010), "Empirical industrial organization: A progress report," Journal of Economic Perspectives 24, 145-?162.

[35] Foster, L., Haltiwanger, J. and C. Syverson (2008), "Reallocation, firm turnover, and efficiency: Selection on productivity or profitability? American Economic Review 98, 394-425.

[36] Fujita, M. and J.-F. Thisse (2013), Economics of Agglomeration. Cities, Industrial Location, and Regional Growth, Second Edition, Cambridge University Press, Cambridge.

[37] Furusawa, T. and H. Konishi (2007), "Free trade networks," Journal of International Economics 72, 310-335.

[38] Gabszewicz, J.J. and J.-F. Thisse (1979), "Price competition, quality and income disparities," Journal of Economic Theory 20, 340-359. 
[39] Gabszewicz, J.J. and J.-F. Thisse (1986), "Spatial competition and the location of firms, Fundamentals of Pure and Applied Economics 5, 1-71.

[40] Glimcher, P.W. and E. Fehr (2013), Neuroeconomics: Decision Making and the Brain, New York: Academic Press.

[41] Goyal, S. and S. Joshi (2003), "Networks of collaboration in oligopoly," Games and Economic Behavior 43, 57-85.

[42] Goyal, S. and J.L. Moraga-Gonzalez (2001), "R\&D networks," RAND Journal of Economics $32,686-707$.

[43] Gur, Y., Saban, D. and N.E. Stier-Moses (2017), "The competitive facility location problem in a duopoly," Unpublished manuscript, Columbia University.

[44] Hart, O.D. (1979), "Monopolistic competition in a large economy with differentiated commodities," Review of Economic Studies 46, 1-30.

[45] Hart, O.D. (1985), "Monopolistic competition in the spirit of Chamberlin: A general model," Review of Economic Studies 52, 529-546.

[46] Helpman, E. and P.R. Krugman (1989), Trade Policy and Market Structure, Cambridge, MA: MIT Press.

[47] Hoberg, G. and G. Phillips (2016), "Text-based network industries and endogenous product differentiation," Journal of Political Economy 124, 1423-1465.

[48] Hotelling, H. (1929), "Stability in competition," Economic Journal 39, 41-57.

[49] Jackson, M.O. (2008), Social and Economic Networks, Princeton: Princeton University Press.

[50] Jackson, M.O. (2014), "Networks in the understanding of economic behaviors," Journal of Economic Perspectives 28, 3-22.

[51] Jackson, M.O., Rogers, B.W. and Y. Zenou (2017), "The economic consequences of social network structure," Journal of Economic Literature 55, 49-95.

[52] Jackson, M.O and A. Wolinsky (1996), "A strategic model of social and economic networks," Journal of Economic Theory 71, 44-74.

[53] Jackson, M.O. and Y. Zenou (2015), "Games on networks, "In: P. Young and S. Zamir (Eds.), Handbook of Game Theory, Vol. 4, Amsterdam: Elsevier, pp. 91-157.

[54] Kamakura, W.A. and G.J. Russell (1989), "A probabilistic choice model for market segmentation and elasticity structure," Journal of Marketing Research 26, 379-391.

[55] König, M., Liu, X. and Y. Zenou (2018), "R\&D networks: Theory, empirics and policy implications", Unpublished manuscript, Monash University. 
[56] Lancaster, K.J. (1966), "A new approach to consumer theory," Journal of Political Economy $74,132-157$.

[57] Lancaster, K.J. (1979), Variety, Equity, and Efficiency: Product Variety in an Industrial Society, New York: Columbia University Press.

[58] Lancaster, K.J (1990), "The economics of product variety: A survey," Marketing Science 9, 189-206.

[59] Matsuyama, K. (1995), "Complementarities and cumulative processes in models of monopolistic competition," Journal of Economic Literature 33, 701-729.

[60] Mayer, T., Melitz, M.J. and G.I.P. Ottaviano (2014), "Market size, competition, and the product mix of exporters," American Economic Review 104, 495-536.

[61] Melitz, M.J. (2003), "The impact of trade on intra-industry reallocations and aggregate industry productivity," Econometrica 71, 1695-1725.

[62] Melitz, M.J. and G.I.P. Ottaviano (2008), "Market size, trade, and productivity," Review of Economic Studies 75, 295-316.

[63] Osharin, A., Thisse, J. F., Ushchev, P., and V. Verbus (2014), "Monopolistic competition and income dispersion," Economics Letters 122, 348-352.

[64] Ottaviano, G.I.P., Tabuchi, T. and J.-F. Thisse (2002), "Agglomeration and trade revisited," International Economic Review 43, 409-436.

[65] Parenti, M ., Thisse, J.-F, and P. Ushchev (2017), "Toward a theory of monopolistic competition," Journal of Economic Theory 167, 86-115.

[66] Perloff, J.M. and S.C. Salop (1985), "Equilibrium with product differentiation," Review of Economic Studies 52, 107-20.

[67] Picard, P.M. and T. Okubo (2012), "Firms' locations under demand heterogeneity," Regional Science and Urban Economics 42, 961-974.

[68] Picard P.M. and T. Tabuchi (2013), "On microfoundations of the city," Journal of Economic Theory 148, 2561-2582.

[69] Rosen, S. (1974), "Hedonic prices and implicit markets: product differentiation in pure competition," Journal of Political Economy 82, 34-55.

[70] Salop, S. C. (1979), "Monopolistic competition with outside goods," Bell Journal of Economics $10,141-156$.

[71] Sattinger, M. (1984), "Value of an additional firm in monopolistic competition," Review of Economic Studies 51, 321-332. 
[72] Shi, M. (2003), "Social network-based discriminatory pricing strategy," Marketing Letters 14, 239-256.

[73] Singh, N. and X. Vives (1984). Price and quantity competition in a differentiated duopoly. RAND Journal of Economics 15, 546-554.

[74] Tarasov, A. (2014), "Preferences and income effects in monopolistic competition models," Social Choice and Welfare 42, 647-669.

[75] Tirole, J. (1988), The Theory of Industrial Organization, Cambridge, MA: MIT Press.

[76] Vives, X. (1999), Oligopoly Pricing. Old Ideas and New Tools, Cambridge, MA: MIT Press.

[77] Wendell, R.E. and R.D. McKelvey (1981), "New prospectives in competition location theory," European Journal of Operational Research 6, 174-182.

[78] Westbrock, B. (2010), "Natural concentration in industrial research collaboration," RAND Journal of Economics 41, 351-371.

[79] Zhelobodko, E., Kokovin, S., Parenti, M., and J.-F. Thisse (2012), "Monopolistic competition: Beyond the constant elasticity of substitution," Econometrica 80, 2765-2784. 


\section{APPENDIX A: Proofs}

Proof of Lemma 1: Let us give a condition that ensures that the utility function (3) is strictly concave. In other words, it must be that $\mathbf{I}+\delta \mathbf{G}$ is a positive definite matrix. A necessary and sufficient condition for this to be true is that all eigenvalues of $\mathbf{I}+\delta \mathbf{G}$ are strictly positive. Since $\mathbf{G}$ is symmetric, then all its eigenvalues are real and we can thus rank them. Since the eigenvalues of $\mathbf{I}+\delta \mathbf{G}$ are equal to: $1+\delta \lambda_{1}(\mathbf{G}), \ldots, 1+\delta \lambda_{N}(\mathbf{G})$, the utility function is strictly concave if and only if (4) holds 17 .

Proof of Proposition 1. Restate $(23)$ as follows:

$$
\widetilde{p}_{i}^{*}=\frac{1}{2}\left(1+\sum_{j \in \mathcal{N}, j \neq i} \frac{b_{i j} \bar{\alpha}_{j}}{b_{i i} \bar{\alpha}_{i}}\left(1-\tilde{p}_{j}\right)\right), \quad i \in \mathcal{N},
$$

where $\widetilde{p}_{i} \equiv p_{i} / \bar{\alpha}_{i}$. Denote by $\widetilde{\mathbf{p}}^{*}$ the linear mapping described by (35).

Condition (20) implies that $\widetilde{\mathbf{p}}^{*}$ is a contraction mapping. Indeed, by (20) we have

$$
\sum_{j \in \mathcal{N}, j \neq i}\left|\frac{b_{i j} \bar{\alpha}_{j}}{b_{i i} \bar{\alpha}_{i}}\right|<1 \quad \text { for all } i \in \mathcal{N},
$$

which is the exact criterion for a linear mapping to be a contraction with respect to the $L_{1}$-norm in $\mathbb{R}^{N}$.

We now show that there exists a convex compact set $\Delta \subset \mathbb{R}_{+}^{N}$, such that (i) $\Delta$ is separated from the origin, and (ii) $\widetilde{\mathbf{p}}^{*}(\Delta) \subseteq \Delta$, i.e. $\widetilde{\mathbf{p}}^{*}$ maps $\Delta$ into itself.

Consider $\Delta \equiv\left\{\widetilde{\mathbf{p}} \in \mathbb{R}_{+}^{N} \mid \varepsilon \leq \widetilde{p}_{i} \leq 1-\varepsilon\right\}$, where $\varepsilon$ is a real satisfying $0<\varepsilon<1 / 2$ and to be further specified below. As implied by (35), the necessary and sufficient condition for $\widetilde{\mathbf{p}}^{*}(\Delta) \subseteq \Delta$ is given by

$$
-(1-2 \varepsilon) \leq \sum_{j \in \mathcal{N}, j \neq i} \frac{b_{i j} \bar{\alpha}_{j}}{b_{i i} \bar{\alpha}_{i}}\left(1-\tilde{p}_{j}\right) \leq 1-2 \varepsilon,
$$

for all $j \in \mathcal{N}$ and for all $\widetilde{p}_{j} \in[\varepsilon, 1-\varepsilon]$.

Let us choose $\varepsilon$ so that the following inequalities hold:

$$
0<\varepsilon<\frac{1}{2}\left(1-\max _{i \in \mathcal{N}} \sum_{j \in \mathcal{N}, j \neq i} \frac{\left|b_{i j}\right| \bar{\alpha}_{j}}{b_{i i} \bar{\alpha}_{i}}\right) .
$$

As implied by the the diagonal dominance condition (20), the right-hand side of (37) is strictly positive and is strictly less $1 / 2$, hence $\Delta$ is non-empty and bounded away from zero. Furthermore, it is readily verified that, for any $\varepsilon$ satisfying (37), the condition $(36)$ also holds. Thus, $\widetilde{\mathbf{p}}^{*}(\Delta) \subseteq \Delta$.

To sum up, $\widetilde{\mathbf{p}}^{*}$ is a contraction mapping that maps the non-empty closed set $\Delta$ into itself, while $\Delta$ is bounded away from the origin. Hence, by the contraction mapping theorem, $\widetilde{\mathbf{p}}^{*}$ has a unique

\footnotetext{
${ }^{17}$ Observe that $\lambda_{N}(\mathbf{G})<0$ since $\operatorname{tr}(\mathbf{G})=\sum_{i=1}^{n} \lambda_{i}(\mathbf{G})=0$
} 
fixed point in $\Delta$. In other words, there exists a unique interior equilibrium price vector $\mathbf{p}^{*}$, such that $p_{i}^{*}<\bar{\alpha}_{i}$ for each $i \in \mathcal{N}$. Since the best reply functions (23) are linear, a closed-form solution for the equilibrium prices $\mathbf{p}^{*}$ given by $(25)$ is easily derived. This completes the proof.

Proof of Proposition 2. Plugging $\mathbf{p}=\mathbf{p}^{*}$ into the first-order conditions $(22)$ and implicitly differentiating these conditions with respect to $\delta$ yields

$$
2 \frac{\partial b_{i i}}{\partial \delta} p_{i}^{*}+2 b_{i i} \frac{\partial p_{i}^{*}}{\partial \delta}+\sum_{j \neq i} \frac{\partial b_{i j}}{\partial \delta} p_{j}^{*}+\sum_{j \neq i} b_{i j} \frac{\partial p_{j}^{*}}{\partial \delta}=\sum_{j \in \mathcal{N}} \frac{\partial b_{i j}}{\partial \delta} \bar{\alpha}_{j} .
$$

Consider first the special case of $\delta=0$. It follows from (22) and (11) that $p_{i}^{*}=\bar{\alpha}_{i} / 2, b_{i i}=1$ and $b_{i j}=0$ for $i \neq j$. Furthermore, (11) implies that $\partial b_{i j} /\left.\partial \delta\right|_{\delta=0}=-g_{i j}$. Thus, for $\delta=0$, (38) amounts to:

$$
\left.\frac{\partial p_{i}^{*}}{\partial \delta}\right|_{\delta=0}=-\frac{1}{4} \sum_{j \in \mathcal{N}} g_{i j} \bar{\alpha}_{j}=-\frac{1}{4} \sum_{j \in \mathcal{N}(i)} \bar{\alpha}_{j}<0 .
$$

Since both sides of (38) are continuously differentiable in $\delta$, it follows from the implicit function theorem that $\partial p_{i}^{*} / \partial \delta$ also are. Hence, $\partial p_{i}^{*} / \partial \delta<0$ must be satisfied when $\delta$ belongs to an open interval $(0, \widehat{\delta}(\phi, \mathbf{G}))$, where $\widehat{\delta}(\phi, \mathbf{G})$ is determined as follows: if there exists a solution (in terms of $\delta)$ to the equation

$$
\max _{i \in \mathcal{N}}\left(\frac{\partial p_{i}^{*}(\phi, \delta, \mathbf{G})}{\partial \delta}\right)=0
$$

within the interval $\left(0,1 / \lambda_{1}(\mathbf{G})\right)$, then $\widehat{\delta}(\phi, \mathbf{G})$ is defined as a minimum of such solutions, otherwise $\widehat{\delta}(\phi, \mathbf{G}) \equiv 1 / \lambda_{1}(\mathbf{G})$. We conclude that $\partial p_{i}^{*} / \partial \delta<0$ whenever $\delta<\widehat{\delta}(\phi, \mathbf{G})$. This completes the proof.

Proof of Proposition 3 . Differentiating 22 with respect to $\phi$ yields

$$
2 b_{i i} \frac{\partial p_{i}^{*}}{\partial \phi}+\sum_{j \neq i} b_{i j} \frac{\partial p_{j}^{*}}{\partial \phi}=\sum_{j \in \mathcal{N}} b_{i j} \frac{\partial \bar{\alpha}_{j}}{\partial \phi}
$$

where

$$
\frac{\partial \bar{\alpha}_{j}}{\partial \phi}=\alpha \sum_{k=0}^{d} k \phi^{k-1} \frac{N_{k}(j)}{N}>0
$$

Evaluating (40) at $\delta=0$ yields

$$
2 \frac{\partial p_{i}^{*}}{\partial \phi}=\frac{\partial \bar{\alpha}_{i}}{\partial \phi}>0
$$

Since both sides of 40 are continuously differentiable in $\delta$, it follows from the implicit function theorem that $\partial p_{i}^{*} / \partial \phi$ also are. Hence, $\partial p_{i}^{*} / \partial \phi>0$ must be satisfied when $\delta$ belongs to an open 
interval $(0, \widetilde{\delta}(\phi, \mathbf{G}))$, where $\widetilde{\delta}(\phi, \mathbf{G})$ is determined as follows: if there exists a solution (in terms of $\delta)$ to the equation

$$
\min _{i \in \mathcal{N}}\left(\frac{\partial p_{i}^{*}(\phi, \delta, \mathbf{G})}{\partial \phi}\right)=0
$$

within the interval $\left(0,1 / \lambda_{1}(\mathbf{G})\right)$, then $\widetilde{\delta}(\phi, \mathbf{G})$ equals the minimum of such solutions, otherwise we set $\widetilde{\delta}(\phi, \mathbf{G}) \equiv 1 / \lambda_{1}(\mathbf{G})$. As a result, we have $\partial p_{i}^{*} / \partial \phi>0$ whenever $\delta<\widehat{\delta}(\phi, \mathbf{G})$.

Proof of Lemma 2. We set $\Delta N i \equiv N_{i}^{\prime}-N_{i}$ for all $i \in \mathcal{N}$. The change of the denominator may be expressed as $\left(\delta+\delta^{2}\right) \Delta r+\mathcal{O}\left(\delta^{3}\right)$, which is positive when $\delta$ is not too large, since the emergence of new links leads to $\Delta r>0$. Thus, it remains to prove that the change in the numerator is also positive. The latter is given by

$$
\phi \Delta r+\phi^{2} \Delta N_{2}+\ldots+\phi^{d} \Delta N_{d}
$$

where $d$ is the diameter of the network $(\mathcal{N}, \mathbf{G}){ }^{18}$ Observe that, since the set of nodes $\mathcal{N}$ remains unchanged, we have $r+N_{2}+\ldots+N_{d}=r^{\prime}+N_{2}^{\prime}+\ldots+N_{d}^{\prime}=N$. This can be restated in terms of changes as follows:

$$
\Delta r+\Delta N_{2}+\ldots+\Delta N_{d}=0 .
$$

Moreover, the following inequalities hold:

$$
\Delta r>0, \quad \Delta r+\Delta N_{2} \geq 0, \quad \ldots, \quad \Delta r+\Delta N_{2}+\ldots+\Delta N_{d-1} \geq 0 .
$$

Indeed, the magnitude $r+N_{2}+\ldots+N_{k}$ shows the number of nodes which are not further than $k$ from a fixed node. Because the distance between any two nodes either decreases or remains unchanged with the emergence of new links, it must be that 42 hold true.

Furthermore, since $\phi \in(0,1)$, using 42 yields

$$
\begin{gathered}
\phi \Delta r+\phi^{2} \Delta N_{2}>\left(\Delta r+\Delta N_{2}\right) \phi^{2}, \\
\phi \Delta r+\phi^{2} \Delta N_{2}+\phi^{3} \Delta N_{3}>\left(\Delta r+\Delta N_{2}\right) \phi^{2}+\phi^{3} \Delta N_{3} \geq\left(\Delta r+\Delta N_{2}+\Delta N_{3}\right) \phi^{3},
\end{gathered}
$$

and so forth. Applying sequentially these inequalities, we end up with

$$
\phi \Delta r+\phi^{2} \Delta N_{2}+\ldots+\phi^{d} \Delta N_{d}>\left(\Delta r+\Delta N_{2}+\ldots+\Delta N_{d}\right) \phi^{d} .
$$

Combining this with 41, we have $\phi \Delta r+\phi^{2} \Delta N_{2}+\ldots+\phi^{d} \Delta N_{d}>0$, which completes the proof.

Proof of Proposition 4. As stated by in Lemma 2, $M A E(\phi)>0$ whenever $\phi \in(0,1)$. What happens when either $\phi=0$ or $\phi=1$ ? Obviously, $M A E(0)=0$. More interestingly, we also have

\footnotetext{
${ }^{18}$ Since $\left(\mathcal{N}, \mathbf{G}^{\prime}\right)$ is obtained from $(\mathcal{N}, \mathbf{G})$ by means of adding new links, we always have $d^{\prime} \leq d$. When $d^{\prime}<d$, we set by definition $N_{k}^{\prime} \equiv 0$ for all $k>d^{\prime}$.
} 
$M A E(1)=0$, which is implied by (41). This means that $M A E(\phi)$ is a non-monotone function of $\phi$ over $[0,1]$. Hence, $\mathrm{d}(M A E) / \mathrm{d} \phi=0$ has at least one internal solution. Denote by $\phi_{0}$ and $\phi_{1}$ the smallest and the largest of such solutions, respectively ${ }^{19}$ Since $M A E(\phi) \geq 0$, and $M A E(\phi)=0$ if and only if $\phi \in\{0,1\}$, it must be that $M A E(\phi)$ increases over $\left[0, \phi_{0}\right]$ and decreases over $\left[\phi_{1}, 1\right]$.

Because the competition effect $C E(\delta)=\left(\delta+\delta^{2}\right) \Delta r+\mathcal{O}\left(\delta^{3}\right)$ is strictly positive, we have $M A E(\phi)<C E(\delta) n p^{*} / \alpha$ when $\phi$ is either close to zero or close to one. However, if $\delta$ is not too large for $C E(\delta) n p^{*} / \alpha<\min _{\phi \in\left[\phi_{0}, \phi_{1}\right]} M A E(\phi)$ to hold, then the equation $C E(\delta) n p^{*} / \alpha=M A E(\phi)$ has a unique internal solution $\underline{\phi}$ over $\left[0, \phi_{0}\right]$, and a unique internal solution $\bar{\phi}$ over $\left[\phi_{1}, 1\right]$. Moreover, $C E(\delta) p^{*}<M A E(\phi)$ for all $\phi \in(\underline{\phi}, \bar{\phi})$, and $C E(\delta) n p^{*} / \alpha>M A E(\phi)$ otherwise.

\footnotetext{
${ }^{19}$ Because $M A E(\phi)$ is a polynomial of $\phi, \mathrm{d} M A E / \mathrm{d} \phi=0$ has a finite number of internal solutions. Hence $\phi_{0}$ and $\phi_{1}$ are always well-defined.
} 


\section{APPENDIX B: Market Segmentation and Product-Variety Networks}

To further clarify the link between market segmentation and product-variety networks, we now consider a space of characteristics. Following Rosen (1974), we assume this space to be (a region in) a finite-dimensional space $\mathbb{R}^{d}$ endowed with the standard Euclidean norm $\|\cdot\|$. Let products $i$ and $j$ be described, respectively, by the combinations $\mathbf{z}_{i}, \mathbf{z}_{j} \in \mathbb{R}^{d}$ of characteristics. Fix some positive real $r>0$, and refer to firms $i$ and $j$ as sharing the same market segment if and only if the characteristics of products they supply are sufficiently close to each other: $\left\|\mathbf{z}_{i}-\mathbf{z}_{j}\right\| \leq r$. Otherwise, firms $i$ and $j$ belong to different market segments. In this context, a product vatiety network over the set $\mathcal{N}=1,2, \ldots, N$ of varieties can be equivalently represented by the $r$-proximity graph of the set $\left\{\mathbf{z}_{1}, \mathbf{z}_{2}, \ldots, \mathbf{z}_{N}\right\} \subset \mathbb{R}^{d}$ of the corresponding bundles of characteristics:

$$
g_{i j}=1 \quad \Longleftrightarrow \quad 0<\left\|\mathbf{z}_{i}-\mathbf{z}_{j}\right\| \leq r .
$$

Assume now that we do not observe the space of characteristics, but we have somehow constructed a network of competitive linkages among firms (say, by estimating strategic complementarities using price data as in Amiti et al. (2016)). The above interpretation of product variety networks allows us to make inference about fundamental structural properties of the underlying space of characteristics, a problem of paramount importance for both hedonic regressions and marketing research. 
ONLINE APPENDIX FOR:

Price Competition in Product Variety Networks

\section{By Philip Ushchev (National Research University Higher School of Economics) and Yves Zenou (Monash University and IFN)}

\section{Specific Network Structures}

\section{Chamberlinian competition}

Consider the Chamberlin-type spatial structure (Figure 2a) with $N$ varieties. In this case, $\mathbf{G}$ has only two distinct eigenvalues, $\lambda_{1}=N-1, \lambda_{2}=\lambda_{3}=\ldots=\lambda_{N}=-1$. Hence, (4) and (8) boil down to $\gamma<1 /(N-1)$.

Consider the special case of $N=4$. Then, if $\delta<1 / 3$, we have:

$$
\mathbf{B}(-\delta, \boldsymbol{G}) \equiv(\mathbf{I}+\delta \boldsymbol{G})^{-1}=\frac{1}{\left(1+2 \delta-3 \delta^{2}\right)}\left(\begin{array}{cccc}
1+2 \delta & -\delta & -\delta & -\delta \\
-\delta & 1+2 \delta & -\delta & -\delta \\
-\delta & -\delta & 1+2 \delta & -\delta \\
-\delta & -\delta & -\delta & 1+2 \delta
\end{array}\right)
$$

and

$$
\alpha_{i k}(\phi, \boldsymbol{G})=\left\{\begin{array}{cl}
\alpha & \text { if } i=k \\
\alpha \phi & \text { if } i \neq k
\end{array}\right.
$$

As a result, the individual demand (say for individual 1) for all four varieties is given by:

$$
\mathbf{x}_{1}^{*}=\frac{1}{\beta\left(1+2 \delta-3 \delta^{2}\right)}\left(\begin{array}{c}
\alpha(1+2 \delta-3 \delta \phi)-(1+2 \delta) p_{1}+\delta\left(p_{2}+p_{3}+p_{4}\right) \\
\alpha(\phi-\delta)-(1+2 \delta) p_{2}+\delta\left(p_{1}+p_{3}+p_{4}\right) \\
\alpha(\phi-\delta)-(1+2 \delta) p_{3}+\delta\left(p_{1}+p_{2}+p_{4}\right) \\
\alpha(\phi-\delta)-(1+2 \delta) p_{4}+\delta\left(p_{1}+p_{2}+p_{3}\right)
\end{array}\right)
$$

As implied by (44), when $\phi \rightarrow 1$, the demand system becomes completely symmetric.

We can now calculate the equilibrium. If $\delta<1 / 3$, then the unique (symmetric) Nash equilibrium is given by:

$$
\begin{gathered}
p_{1}^{*}=p_{2}^{*}=p_{3}^{*}=p_{4}^{*}=\frac{\alpha(1-\delta)(3 \phi+4)}{4(\delta+2)} \\
X_{1}^{*}=X_{2}^{*}=X_{3}^{*}=X_{4}^{*}=\frac{\alpha}{\beta} \frac{(2 \delta+1)(3 \phi+4)}{3 \delta^{2}+7 \delta+2}
\end{gathered}
$$

Chen-Riordan competition Consider now the Chen-Riordan spatial structure (Figure 2d) with $N$ varieties, the eigenvalues of are: $\lambda_{1}=\sqrt{N-1}, \quad \lambda_{2}=\ldots=\lambda_{N-1}=0, \lambda_{N}=-\sqrt{N-1}$. Hence, a necessary and sufficient condition for (4) and (8) to hold is $\delta<1 / \sqrt{N-1}$. 
For $N=4$ and $\delta<1 / \sqrt{3}$, we have ( 1 is the star variety):

$$
\mathbf{B}(-\delta, \boldsymbol{G})=\frac{1}{\left(1-3 \delta^{2}\right)}\left(\begin{array}{cccc}
1 & -\delta & -\delta & -\delta \\
-\delta & 1-2 \delta^{2} & \delta^{2} & \delta^{2} \\
-\delta & \delta^{2} & 1-2 \delta^{2} & \delta^{2} \\
-\delta & \delta^{2} & \delta^{2} & 1-2 \delta^{2}
\end{array}\right)
$$

and

$$
\alpha_{i 1}(\phi, \boldsymbol{G})=\left\{\begin{array}{cc}
\alpha & \text { if } i=1 \\
\alpha \phi & \text { if } i \neq 1
\end{array}\right.
$$

while for $k \neq 1$,

$$
\alpha_{i k}(\phi, \boldsymbol{G})=\left\{\begin{array}{cc}
\alpha & \text { if } i=k, \\
\alpha \phi & \text { if } i=1, \\
\alpha \phi^{2} & \text { otherwise }
\end{array}\right.
$$

As a result, the individual demand for individual 1 (whose ideal variety is the star variety) for all four varieties is given by:

$$
\mathbf{x}_{1}^{*}=\frac{1}{\beta\left(1-3 \delta^{2}\right)}\left(\begin{array}{c}
\alpha(1-3 \delta \phi)-p_{1}+\delta\left(p_{2}+p_{3}+p_{4}\right) \\
\alpha(\phi-\delta)-\left(1-2 \delta^{2}\right) p_{2}+\delta p_{1}-\delta^{2}\left(p_{3}+p_{4}\right) \\
\alpha(\phi-\delta)-\left(1-2 \delta^{2}\right) p_{3}+\delta p_{1}-\delta^{2}\left(p_{2}+p_{4}\right) \\
\alpha(\phi-\delta)-\left(1-2 \delta^{2}\right) p_{4}+\delta p_{1}-\delta^{2}\left(p_{2}+p_{3}\right)
\end{array}\right)
$$

The individual demand for all four varieties of a peripheral consumer, say individual 2, whose ideal variety is variety 2 , is given by:

$$
\mathbf{x}_{2}^{*}=\frac{1}{\beta\left(1-3 \delta^{2}\right)}\left(\begin{array}{c}
\alpha\left(\phi-2 \delta \phi^{2}-\delta\right)-p_{1}+\delta\left(p_{2}+p_{3}+p_{4}\right) \\
\alpha\left(1+2 \delta^{2} \phi^{2}-2 \delta^{2}-\delta \phi\right)-\left(1-2 \delta^{2}\right) p_{2}+\delta p_{1}-\delta^{2}\left(p_{3}+p_{4}\right) \\
\alpha\left(\delta^{2}-\delta^{2} \phi^{2}+\phi^{2}-\delta \phi\right)-\left(1-2 \delta^{2}\right) p_{3}+\delta p_{1}-\delta^{2}\left(p_{2}+p_{4}\right) \\
\alpha\left(\delta^{2}-\delta^{2} \phi^{2}+\phi^{2}-\delta \phi\right)-\left(1-2 \delta^{2}\right) p_{4}+\delta p_{1}-\delta^{2}\left(p_{2}+p_{3}\right)
\end{array}\right) .
$$

If $\delta<1 / \sqrt{3}$, then the unique Nash equilibrium is given by:

$$
\left(\begin{array}{c}
p_{1}^{*} \\
p_{2}^{*} \\
p_{3}^{*} \\
p_{4}^{*}
\end{array}\right)=\frac{\alpha}{4\left(4-7 \delta^{2}\right)}\left(\begin{array}{c}
2+6 \phi-3 \delta-5 \delta^{2}+6 \delta^{3}-3 \delta \phi\left(1+5 \delta-4 \delta \phi-2 \delta^{2}+2 \phi\right) \\
2+2 \phi-\delta+4 \phi^{2}-3 \delta^{2}-3 \delta \phi(1+\delta+2 \delta \phi) \\
2+2 \phi-\delta+4 \phi^{2}-3 \delta^{2}-3 \delta \phi(1+\delta+2 \delta \phi) \\
2+2 \phi-\delta+4 \phi^{2}-3 \delta^{2}-3 \delta \phi(1+\delta+2 \delta \phi)
\end{array}\right)
$$

and

$$
\left(\begin{array}{c}
X_{1}^{*} \\
X_{2}^{*} \\
X_{3}^{*} \\
X_{4}^{*}
\end{array}\right)=\frac{\alpha / \beta}{\left(4-19 \delta^{2}+21 \delta^{4}\right)}\left(\begin{array}{c}
2+6 \phi-3 \delta-5 \delta^{2}+6 \delta^{3}-3 \delta \phi\left(1-4 \delta^{2} \phi-2 \delta^{2}+5 \delta+2 \phi\right) \\
\left(1-2 \delta^{2}\right)\left[2+2 \phi-\delta+4 \phi^{2}-3 \delta^{2}-3 \delta \phi(1+\delta+2 \delta \phi)\right] \\
\left(1-2 \delta^{2}\right)\left[2+2 \phi-\delta+4 \phi^{2}-3 \delta^{2}-3 \delta \phi(1+\delta+2 \delta \phi)\right] \\
\left(1-2 \delta^{2}\right)\left[2+2 \phi-\delta+4 \phi^{2}-3 \delta^{2}-3 \delta \phi(1+\delta+2 \delta \phi)\right]
\end{array}\right)
$$


It can easily be verified that, when $\delta$ is not too large, the price and the aggregate demand of the star (central) variety is higher than that of the others.

\section{Comparing Chamberlinian and Chen-Riordan competition}

Let us compare the individual demands between the two types of competition. First, for the Chen-Riordan competition (start network), the network-specific effect on the individual demand, that is the price effect, is the same for all consumers (star and peripheral) in the network as can be seen by the terms after the intercept in the individual demands determined above. We see that (i) own price effect, $\partial x_{i k}^{*} / \partial p_{i}$, is always negative for the demand of each consumer and for each variety, (ii) the cross-price effects, $\partial x_{i k}^{*} / \partial p_{j}, j \neq i$, are positive (negative) if and only if either $i$ or $j$ is the star (both $i$ and $j$ are peripheral varieties). In other words, any peripheral variety is a gross substitute for the star (central) variety (positive effect) while peripheral varieties come to be gross complements to each other (negative effect). This is very different from what we find in the Chamberlinian type of product space (see (44)) where all varieties are direct substitutes to each other: $\partial x_{i k}^{*} / \partial p_{j}=\delta>0, j \neq i$.

Second, consider the intercept in the demand function, which is individual specific and depends on the position in the network. We can see that, for the Chen-Riordan competition, for the star variety, which is at distance 1 to every other variety, the consumer's demand is much higher than for any peripheral variety. This does not imply, however, that the "star" firm $i=1$ will always enjoy more monopoly power than any peripheral firm.

\section{More comparative-statics exercises: Numerical simulations}

\subsection{Impact of $\delta$ on equilibrium prices}

To evaluate the effect of $\delta$ on equilibrium price, we perform some numerical simulations for the line or chain network (Hotelling competition) with $N=5$. This network starts at node $i=1$ and

finishes at node $i=5$, where node 3 is the midpoint, nodes 1 and 5 are the endpoints and nodes 2 and 4 are the between nodes. The results are displayed in Figure A.1. We find results similar to that of Figure 4. Indeed, we see that there is a threshold value of $\delta$ (roughly $\delta=0.2$ ) for which prices of the endpoint nodes become higher than that of the midpoint nodes. This again highlights the trade off between better access to market and facing tougher competition mentioned above. 
Figure A1: Changes in equilibrium prices in the Hotelling network for $N=5$ and $\phi=0.85$

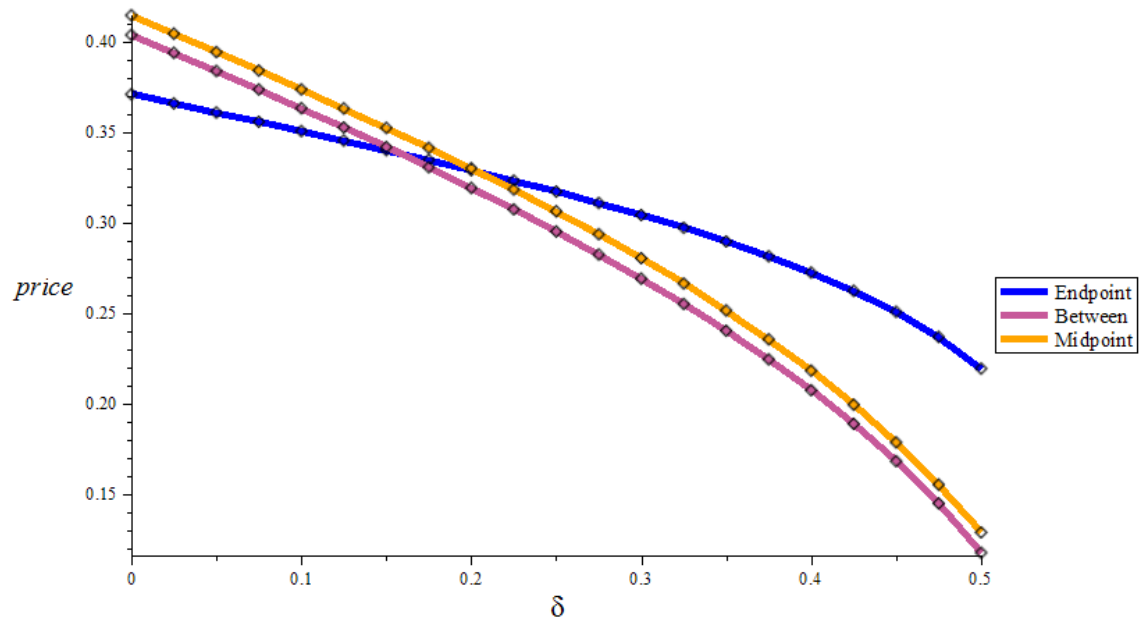

\subsection{Impact of $\phi$ on equilibrium prices}

First, we study the impact of $\phi$ on the equilibrium prices for the Chen-Riordan network for $N=6$ and $\delta=0.3$. The results are displayed in Figure A.2.

Figure A2: Changes in equilibrium prices in the Chen-Riordan network for $N=6$ and $\delta=0.3$

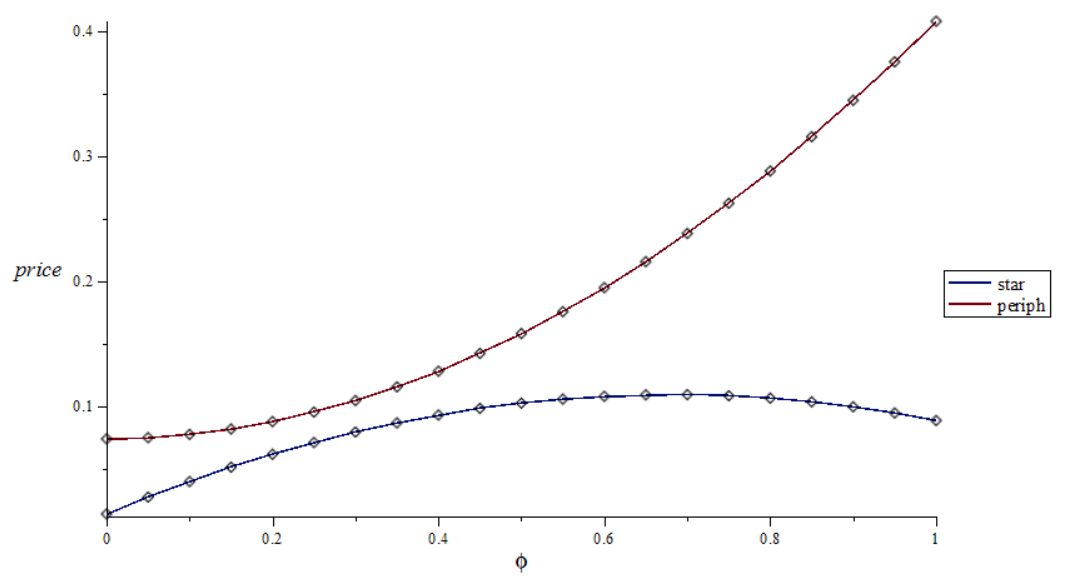

When $\delta=0.3$, being a "star" is even less of an advantage. Indeed, we see that the firm located at the center always charges a lower price than the "peripheral" firms. On top of it, we have here an example of non-monotone behavior of equilibrium prices with respect to $\phi$, which confirms our results above, which hold when $\delta$ is "not too large".

Second, we study the impact of $\phi$ on the equilibrium prices when for the Hotelling network for $N=5$ and $\delta=0.15$. This network starts at node $i=1$ and finishes at node $i=5$, where node 3 is the midpoint, nodes 1 and 5 are the endpoints and nodes 2 and 4 are the between nodes. The results are displayed in Figure A.3. 
Figure A3: Changes in equilibrium prices in the Hotelling network for $N=5$ and $\delta=0.15$

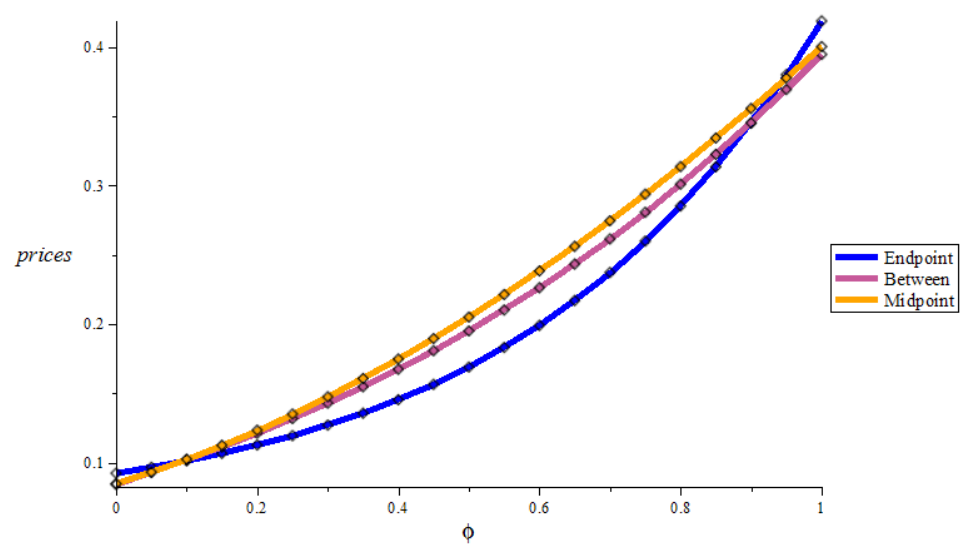

We find results similar to that of Figure 5, even though the picture is more complex. There is a non-monotonic relationship when $\phi$ increases and the mid-point firm tends to charge the highest prices when $\phi$ has intermediate values. When comparing the between nodes and the endpoint nodes, we see again that the former charge a higher prices only when $\phi$ takes intermediate values. This again highlights the trade off between better access to market and facing tougher competition mentioned above.

\section{Asymmetric price equilibrium under a regular network structure}

Assume that $N=12$ and that the product-variety network is described by the Frucht graph, which is a regular graph with $r=3, N=12$, a total of 18 edges. Figure A4 displays the Frucht graph.

Figure A4: Frucht graph

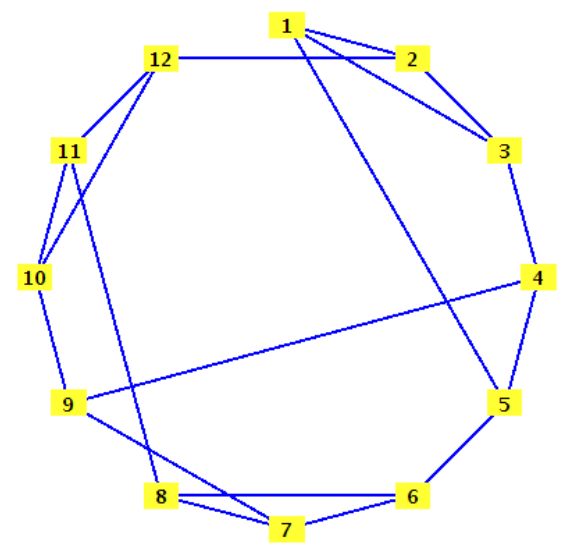

If $(\mathcal{N}, \mathbf{G})$ is the Frucht graph, direct computation under $\beta=1, \delta=0.15$, and $\phi=0.75$ yields 
the following equilibrium price vector:

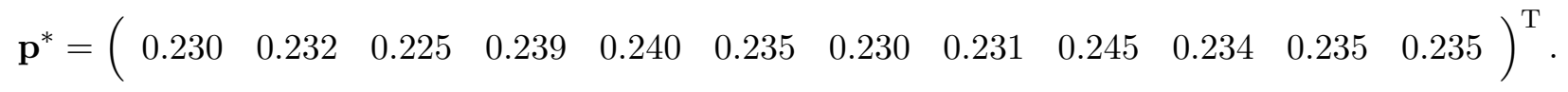

Clearly, this equilibrium is not symmetric, although the network is regular.

\section{Welfare effects when the network becomes denser: Numerical simulations}

Consider three standard networks: the chain network a la Hotelling (Figure 2b), the circular network a la Salop (Figure 2c) and the star-shaped network a la Chen-Riordan (Figure 2d) and compare them to the complete network a la Chamberlin (Figure 2a). Clearly Chamberlin is the densest network (density equal to 1 ) while Salop is the next dense network (density equal to $2 /(n-1))$ and the two other networks have the same lowest density equal to $2 / n$. In the main text, we determined the increase in welfare following an increase in $\phi$ when comparing Chamberlin to Hotelling (Figure 5). Now, we perform the same exercise by comparing Chamberlin to Salop (Figure A5) and to Chen-Riordan (Figure A6).

Figure A5: Chamberlin versus Salop: Welfare effects when $N=6$ and $\delta=0.15$

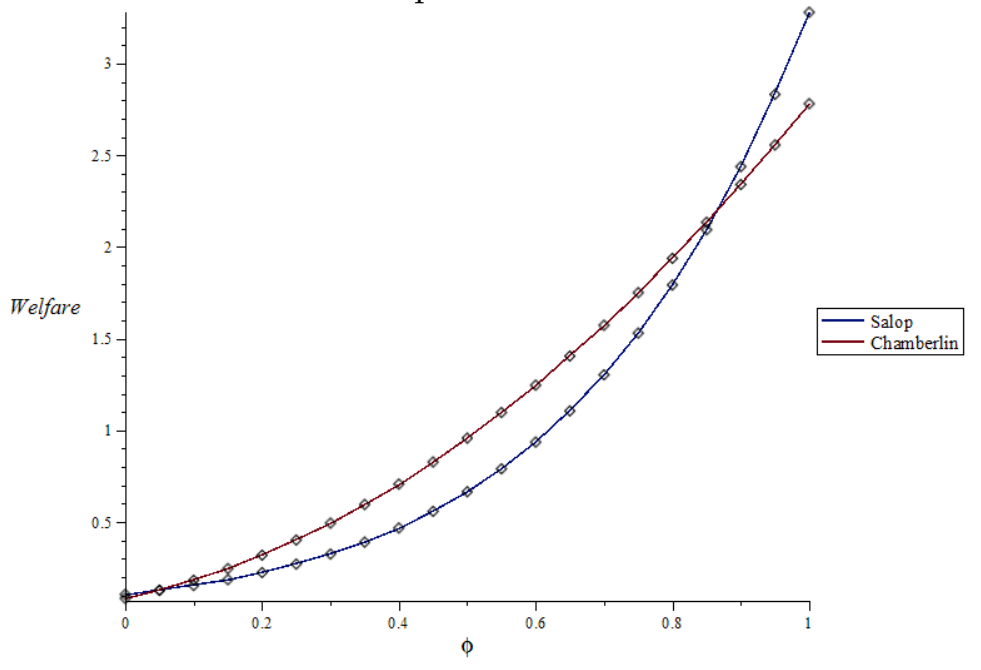


Figure A6: Chamberlin versus Chen-Riordan: Welfare effects when $N=6$ and $\delta=0.15$

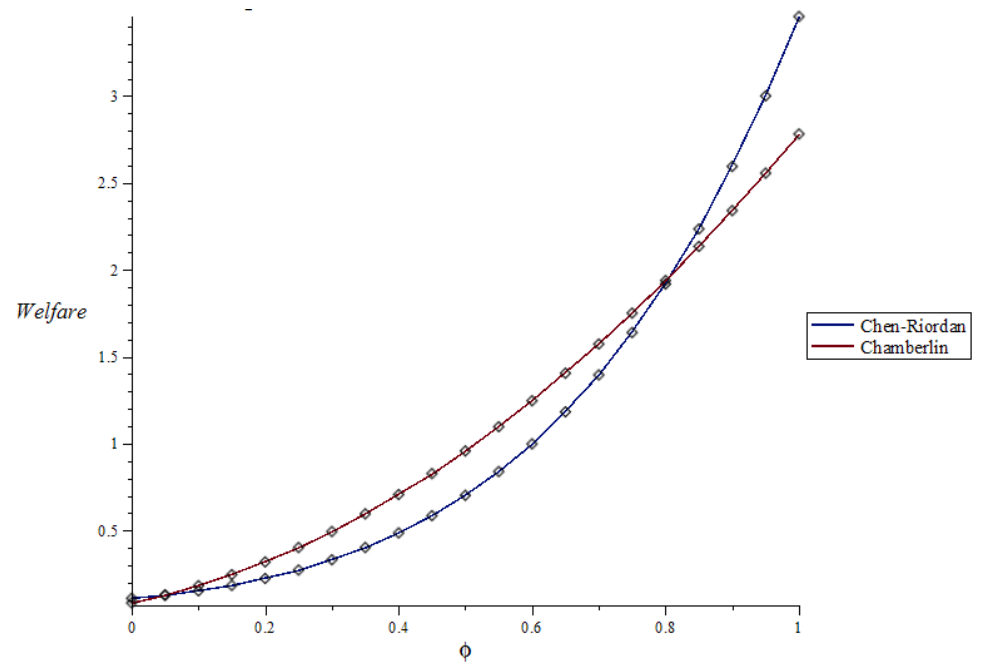

We obtain similar results as in Figure 5, that is when the spatial discount factor $\phi$ is below some threshold level (which is between 0.8 and 0.9 in all the three cases), adding more links is beneficial to the economy from a welfare perspective, whereas, when $\phi$ exceeds this threshold and becomes sufficiently close to one, i.e. when the proximity of all consumers to all varieties is very high, a denser network leads to welfare losses. 